\title{
Evolution of polycyclic basement complexes in the Araçuaí Orogen, based on U-Pb SHRIMP data: Implications for Brazil-Africa links in Paleoproterozoic time ${ }^{\text {th }}$
}

\author{
Carlos M. Noce ${ }^{\mathrm{a}, *}$, Antônio Carlos Pedrosa-Soares ${ }^{\mathrm{a}}$, Luiz Carlos da Silva ${ }^{\mathrm{b}}$, \\ Richard Armstrong ${ }^{\mathrm{c}}$, Danielle Piuzana ${ }^{\mathrm{a}}$ \\ a CPMTC-Instituto de Geociências, Universidade Federal de Minas Gerais, Belo Horizonte (MG) 31270-901, Brazil \\ ${ }^{\mathrm{b}}$ CPRM-Serviço Geológico do Brasil, SGAN 603, $1^{\circ}$ Andar, Brasília (DF) 70830-030, Brazil \\ ${ }^{c}$ Research School of Earth Sciences, The Australian National University, Canberra, ACT 0200, Australia
}

Received 30 November 2006; received in revised form 22 May 2007; accepted 7 June 2007

\begin{abstract}
Paleoproterozoic basement of the Araçuaí Orogen is composed of amphibolite and granulite facies orthogneiss units known as Mantiqueira and Juiz de Fora complexes. Six U-Pb SHRIMP crystallization ages for the Mantiqueira Complex range from $2137 \pm 19$ to $2041 \pm 7 \mathrm{Ma}$. Studied samples are characterized by the abundance of inherited Archean zircon grains, $T_{\mathrm{DM}}$ model ages ranging from 2.9 to $3.2 \mathrm{Ga}$, and strongly negative $\varepsilon_{\mathrm{Nd}(\mathrm{t})}(-9$ to -13$)$. Gneiss protholits were mainly generated by partial melting of older continental material, and the Mantiqueira Complex is related to active margin and syn-collisional magmatism. High-grade gneiss samples of the Juiz de Fora Complex yields crystallization ages of $2119 \pm 16$ and $2084 \pm 13$, and inherited zircon grains are absent. This plutonic unit much probably evolved within an oceanic magmatic arc setting, or on a very stretched continental crust.

The Mantiqueira and Juiz de Fora complexes, together with the Kimezian basement of the West Congo Belt, were parts of a Paleoproterozoic orogenic system disrupted and deeply reworked during the evolution of the Araçuaí-West Congo Orogen. Neoproterozoic metamorphic overprint is dated at ca. 590-574 Ma.
\end{abstract}

(c) 2007 Elsevier B.V. All rights reserved.

Keywords: Paleoproterozoic basement; Gneiss complexes; Araçuaí Orogen; Zircon SHRIMP ages

\section{Introduction}

The Araçuaí Orogen (southeastern Brazil) and the West Congo Belt (southwestern Africa) are counterparts of a Brasiliano-Pan African orogenic system developed between the São Francisco and Congo cratons, during the assembly of Gondwanaland in late Neoproterozoic

\footnotetext{
Contribution to IGCP-509.

* Corresponding author. Tel.: +55 3134994358 ; fax: +553134994440 .

E-mail address: noce@ufmg.br (C.M. Noce).
}

time (Fig. 1). From the oldest evidence of the precollisional stage to the latest post-collisional processes, orogeny lasted approximately from 630 to $490 \mathrm{Ma}$ in the Araçuaí Orogen. The metamorphic peak related to the syn-collisional stage took place at ca. 585-560 Ma (Pedrosa-Soares et al., 2001; Tack et al., 2001; Silva et al., 2005a; Alkmim et al., 2006; Frimmel et al., 2006).

Basement units in the Araçuaí Orogen are essentially composed of orthogneiss complexes of Archean and Paleoproterozoic age. These complexes are exposed in the external (western) and internal (eastern) tectonic domains of the Araçuaí Orogen (Fig. 1). The 

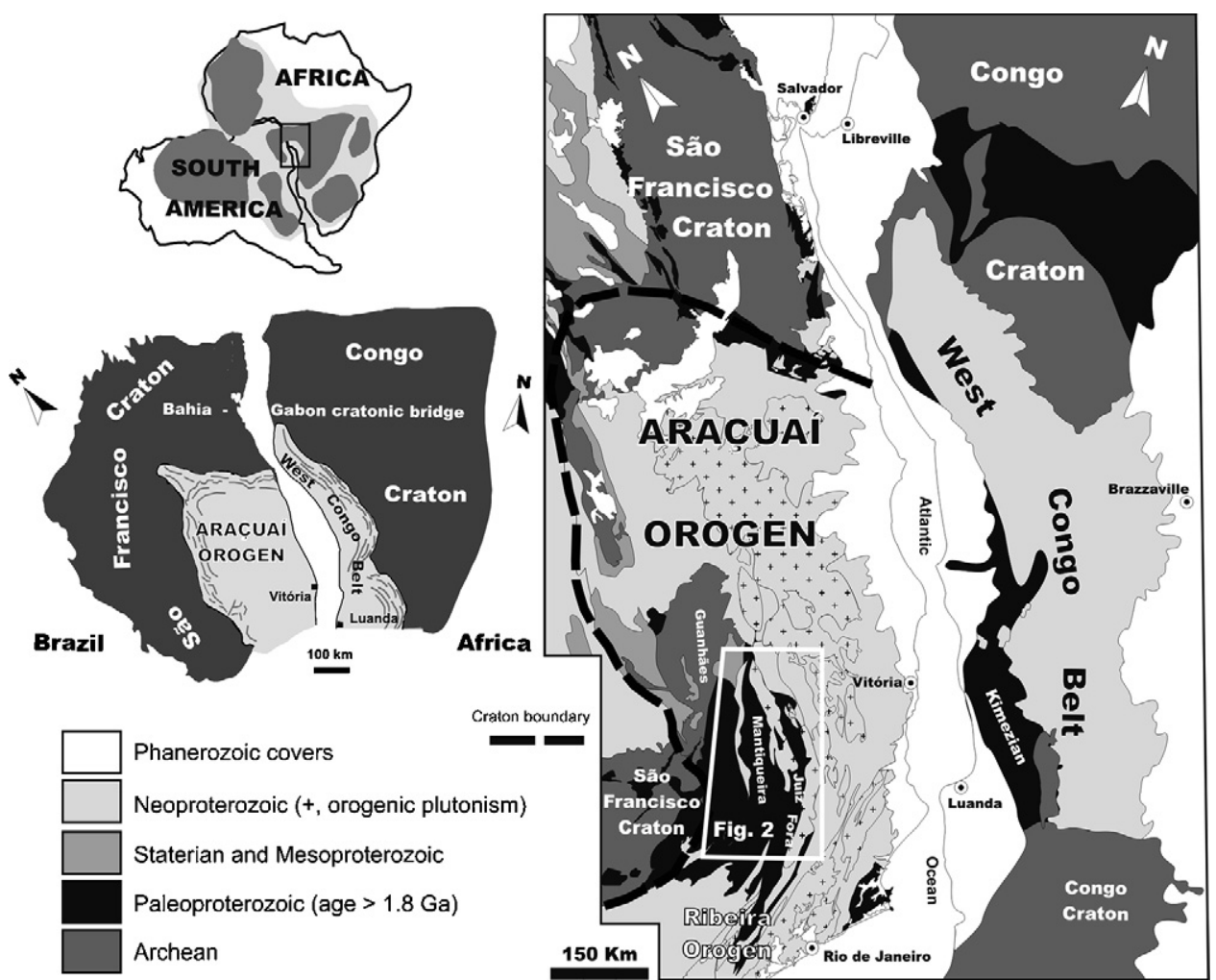

Fig. 1. The Araçuaí-West Congo Orogen and the adjacent São Francisco-Congo craton in the context of West Gondwana (after Alkmim et al., 2006). Geological map of the orogen showing the distribution of basement units (modified from DeWit et al., 1988; Pedrosa-Soares et al., 2001; Tack et al., 2001; Delgado et al., 2003; Silva et al., 2005a).

external domain is the low metamorphic grade, foldand-thrust belt bordering the São Francisco Craton. The internal domain is the high-grade core of the Araçuaí Orogen, where the pre-collisional magmatic arc and widespread syn-collisional granites are found, and regional metamorphism reached the granulite facies. Indeed, such distinct locations of basement complexes in the Araçuaí Orogen imply in different intensities of tectonic reworking and isotopic resetting of the Archean and Paleoproterozoic rocks during the Brasiliano orogeny.

The basement of the Araçuaí Orogen evolved during the assembly of the São Francisco-Congo landmass that probably was a part of a larger Paleoproterozoic continent (e.g., Atlantica Paleocontinent, Rogers and Santosh, 2004). The ancient São Francisco and Congo blocks were stitched together by a Paleoproterozoic orogenic system, called Itabuna-Salvador-Curaça, in Brazil (Barbosa and Sabaté, 2004), or Eburnean, in Africa (Ledru et al., 1994). Absence of Neoproterozoic orogeny allowed the reconstruction of this Paleoproterozoic orogenic system along the cratonic connection on both sides of the Atlantic Ocean, the Bahia-Gabon bridge (Fig. 1): a cratonic link that lasted from ca. 2 Ga up to the Meso- zoic (Porada, 1989; Ledru et al., 1994; Trompette, 1994; Brito Neves et al., 1999; Barbosa and Sabaté, 2004; D'Agrella Filho et al., 2004; Alkmim et al., 2006). The same task, i.e., to reconstruct the Paleoproterozoic orogenic system is much more difficult when dealing with the disrupted and deeply reworked basement remnants within the Araçuaí Orogen (Alkmim and Marshak, 1998; Noce et al., 2000; Teixeira et al., 2000; Silva et al., 2005a). Contributions for achieving this task must consider the correlations between basement units of the West Congo Belt and Araçuaí Orogen, as we discuss afterwards.

This paper focus on the polycyclic evolution of basement complexes of the Araçuaí Orogen, based on $\mathrm{U}-\mathrm{Pb}$ SHRIMP and Sm-Nd data. Eight samples of amphibolite and granulite facies orthogneisses were analysed through the U-Pb SHRIMP method, disclosing a complex and protracted magmatic and metamorphic evolution prior to the Neoproterozoic tectonic reworking. These samples are from the Mantiqueira and Juiz de Fora complexes. We also discuss possible correlations with the basement of the West Congo Belt, and suggest a model for the evolution of the Paleoproterozoic orogenic system. 


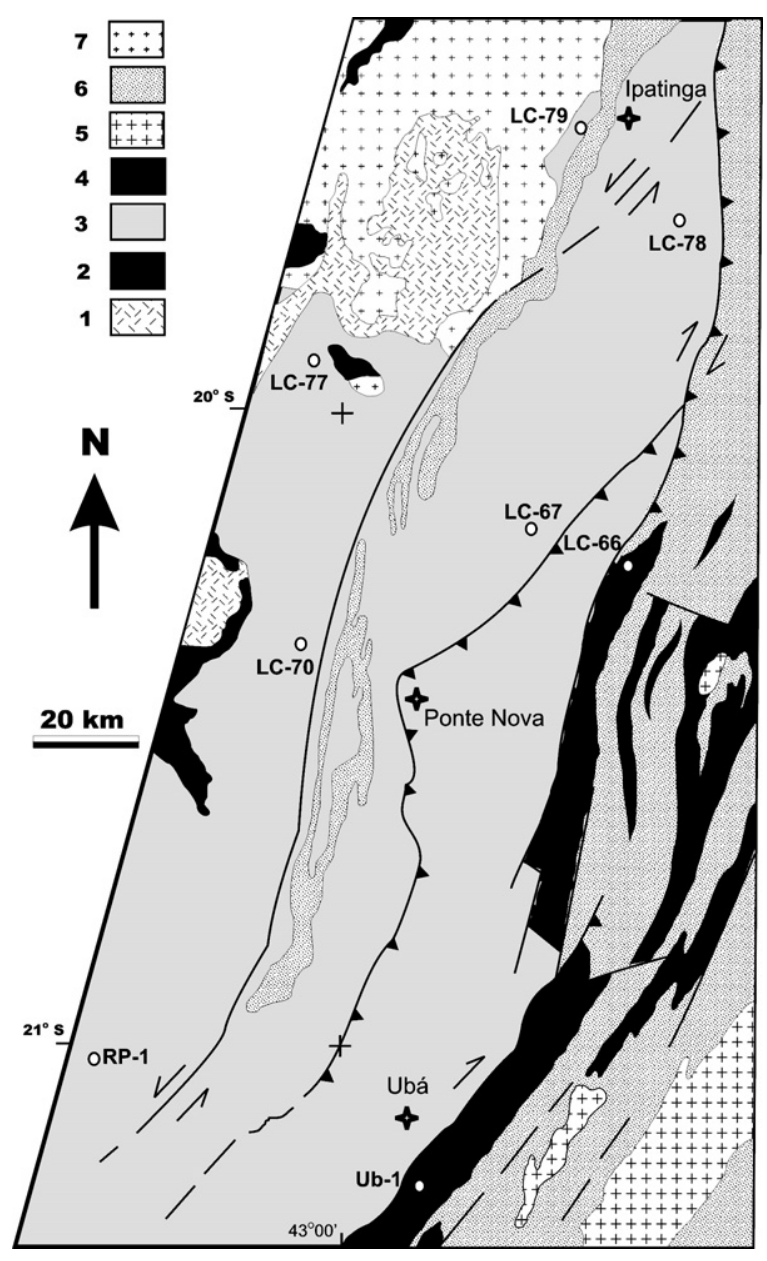

Fig. 2. Geological map of part of the Araçuaí Orogen (see location in Fig. 1), showing the distribution of the Mantiqueira and Juiz de Fora complexes, and sample location (modified from Peres et al., 2004). Archean gneissic complexes (1) and supracrustal rocks (2); Mantiqueira Complex (3); Juiz de Fora Complex (4); Brasiliano units: granitoids (5) and supracrustal rocks (6); Borrachudos Suite (7).

\section{Basement of Araçuaí Orogen}

Two of the basement units of the Araçuaí Orogen, the Guanhães and Mantiqueira gneissic complexes (Fig. 2), consist of large autochthonous and parautochthonous gneissic units that can be traced into the cratonic domain. They are therefore considered to be the reworked margin of the São Francisco Craton, now located in the Araçuaí Orogen. The Guanhães Complex is part of the Archean cratonic nuclei that was bordered by a marginal Paleoproterozoic orogen (Teixeira et al., 2000). The Araçuaí Orogen transects the former Archean-Paleoproterozoic boundary and the Mantiqueira Complex is envisaged as a deeply reworked fragment of a continental magmatic arc. Another Paleoproterozoic basement unit is the Juiz de Fora Complex (Fig. 2), made up of tectonic slices of granulitic orthogneiss and amphibolite. The Mantiqueira and Juiz de Fora complexes were juxtaposed by a dextralreverse, high-angle shear zone of Brasiliano age (the Abre Campo Fault).

The Mantiqueira Complex consists predominantly of banded biotite-amphibole orthogneiss. The thickness of the alternating light and dark bands varies from centimetric to metric, and concordant amphibolite boudins, lenses and layers are very common. The gneissic banding can be explained either by migmatization and/or by intense deformation of distinct magmatic protholits, where tectonism erased any original discordant contacts between individual bodies and stretched them into concordant layers. Regional gneissic banding/foliation trends N-S to NNE-SSW and is parallel to the Brasiliano structures, resulting from intense folding and transposition of an earlier banded structure.

Mantiqueira gneisses are tonalitic to granitic in composition and make up calc-alkaline suites, whereas basic layers and enclaves belong to transitional basaltic series (Duarte et al., 2004). Geochemical modeling, Sr and $\mathrm{Nd}$ signatures suggest that the gneiss protholits were dominantly generated by partial melting of crustal material, and that the Mantiqueira Complex evolved at a continental magmatic arc setting (Duarte et al., 2004, 2005). Remnants of supracrustal rocks within the Mantiqueira Complex are very subordinate and mainly represented by calc-silicatic and quartzite xenoliths (Figueiredo and Teixeira, 1996).

The Juiz de Fora Complex occurs as large tectonic lenses intercalated with Neoproterozoic paragneisses. It is composed of granulite facies orthogneisses, with predominance of enderbitic and minor charnockitic compositions. These rocks make up calc-alkaline suites of juvenile affinity (Figueiredo and Teixeira, 1996; Duarte et al., 1997; Fischel et al., 1998). Lens-shaped bodies of various sizes composed of basic granulites are also present in the Juiz de Fora Complex, and are characterized by Costa (1998) as low-K tholeiites similar to island-arc and ocean floor basalts.

\subsection{Previous geochronological data}

U-Pb SHRIMP ages for the Mantiqueira Complex are in the 2180-2058 Ma time range (Silva et al., 2002), and an age of $2203 \pm 17 \mathrm{Ma}$ obtained through the Laser Ablation-MC-ICPMS method is reported by Heilbron et al. (2001). Available U-Pb ages for enderbite and charnockite gneiss of the Juiz de Fora Complex are $2134 \mathrm{Ma}$ (Machado et al., 1996), 2127 $\pm 33 \mathrm{Ma}$ 
(Heilbron et al., 2001), and $2195 \pm 15 \mathrm{Ma}$ (Silva et al., 2002).

The polycyclic evolution of Paleoproterozoic basement units of the Araçuaí Orogen was highlighted by these previous geochronological studies. SHRIMP U-Pb geochronology showed that those highly transposed, amphibolite and granulite facies orthogneisses had been accreted to, and modified in, several magmatic and metamorphic events (Silva et al., 2002; Silva et al., 2005a). The oldest components intruded in a magmatic arc setting at ca. 2200-2100 Ma. The syn-collisional phase took place between 2100 and $2050 \mathrm{Ma}$, which is the approximate age of the first metamorphic event $\left(\mathrm{M}_{1}\right)$, dated on several metamorphic overgrowths on zircon grains from the older orthogneisses (indicating high temperatures were reached during $\mathrm{M}_{1}$ ), and on magmatic zircons from syn-collisional plutons (Silva et al., 2002, 2005a). A second, and better constrained, metamorphic overprinting $\left(\mathrm{M}_{2}\right)$, corresponding to the Neoproterozoic collage of the Araçuaí Orogen onto the eastern São Francisco cratonic margin, yielded U-Pb SHRIMP metamorphic ages at $580-570 \mathrm{Ma}$ also obtained on zircon metamorphic overgrowths.

\section{Sample descriptions}

The isotopic analyses were performed on six samples of amphibolite facies gneiss from the Mantiqueira Complex, and two samples of granulite facies gneiss from the Juiz de Fora Complex.

Mantiqueira gneisses display granoblastic textures in this section, and main mineral constituents are quartz, microcline, plagioclase, hornblende and biotite. Zircon, titanite, apatite and opaque minerals are the common accessory minerals, plus secondary minerals like chlorite, sericite, epidote and carbonate. On the R1-R2 diagram (after De la Roche et al., 1980) gneiss samples fall mainly on the granodiorite field (LC-67, LC-70,
LC-77, LC-78). Sample RP-1 is tonalitic and sample LC79 is granitic in composition (Table 1). A description of sampled outcrops of Mantiqueira Complex is given bellow:

- Sample RP-1. Medium-grained migmatized gneiss displaying a well-developed centimetric banding, in some places tightly folded. Banded structure is characterized by alternating plagioclase-rich layers and dark layers rich in mafic minerals (biotite and hornblende). Mafic rocks (amphibolites) make up concordant bands and some large disrupted bodies (Fig. 3a and b). Leucossome veins are both concordant and discordant to the rock banding. Collected sample of the banded gneiss did not included leucossome veins, and is tonalitic in composition.

- Sample LC-70. Banded biotite granodiorite gneiss, folded and strongly sheared; sample was taken from a homogeneous light band about $10 \mathrm{~cm}$ thick.

- Sample LC-77. Relatively homogeneous fine-grained biotite granodiorite gneiss with minor hornblende, exhibiting a thin banding.

- Sample LC-79. Complex-folded migmatized gneiss exhibiting alternating banded and more massive layers, plus concordant amphibolite enclaves. Sample was collected from the massive layers. It is mediumto coarse-grained, leucocratic, with biotite and hornblende as mafic minerals, and granitic in composition.

- Sample LC-78. Fine-grained biotite granodiorite gneiss, relatively homogeneous and displaying a welldeveloped banding characterized by alternating light and dark layers that are few millimeters in thickness. Amphibolite enclaves, disrupted and concordant to the rock banding, are also present.

- Sample LC-67. This is a massive, hornblende-biotite granodiorite gneiss, only locally exhibiting a poorly developed banding (Fig. 3c).

Table 1

Major element data for studied samples

\begin{tabular}{|c|c|c|c|c|c|c|c|c|c|c|c|}
\hline Sample & $\mathrm{SiO}_{2}$ & $\mathrm{Al}_{2} \mathrm{O}_{3}$ & $\mathrm{Fe}_{2} \mathrm{O}_{3(\mathrm{t})}$ & $\mathrm{MnO}$ & $\mathrm{MgO}$ & $\mathrm{CaO}$ & $\mathrm{Na}_{2} \mathrm{O}$ & $\mathrm{K}_{2} \mathrm{O}$ & $\mathrm{TiO}_{2}$ & LOI & Total \\
\hline \multicolumn{12}{|c|}{ Mantiqueira Complex } \\
\hline RP-1 & 63.6 & 14.7 & 7.7 & 0.09 & 2.81 & 3.41 & 2.97 & 2.86 & 1.37 & 0.47 & 99.98 \\
\hline LC-70 & 68.8 & 14.7 & 5.24 & 0.04 & 2.99 & 2.29 & 2.37 & 2.22 & 0.36 & 0.32 & 99.33 \\
\hline LC-77 & 68.9 & 18.8 & 2.39 & 0.02 & 2.25 & 1.32 & 2.62 & 2.79 & 0.28 & 0.11 & 99.48 \\
\hline LC-79 & 69.1 & 15.1 & 1.87 & 0.02 & 2.57 & 2.54 & 3.55 & 4.48 & 0.21 & 0.13 & 99.57 \\
\hline LC-78 & 67.7 & 17.4 & 3.99 & 0.05 & 1.54 & 2.45 & 2.6 & 2.83 & 0.55 & 0.32 & 99.43 \\
\hline LC-67 & 69.8 & 14.1 & 4.5 & 0.06 & 2.38 & 1.86 & 2.44 & 3.54 & 0.49 & 0.32 & 99.49 \\
\hline \multicolumn{12}{|c|}{ Juiz de Fora Complex } \\
\hline LC-66a & 53.8 & 17.9 & 9.61 & 0.14 & 3.68 & 7.05 & 2.78 & 2.46 & 1.09 & 0.79 & 99.3 \\
\hline Ub-1 & 67.4 & 14.9 & 4.78 & 0.04 & 2.25 & 3.89 & 2.58 & 2.41 & 0.59 & 0.31 & 99.15 \\
\hline
\end{tabular}



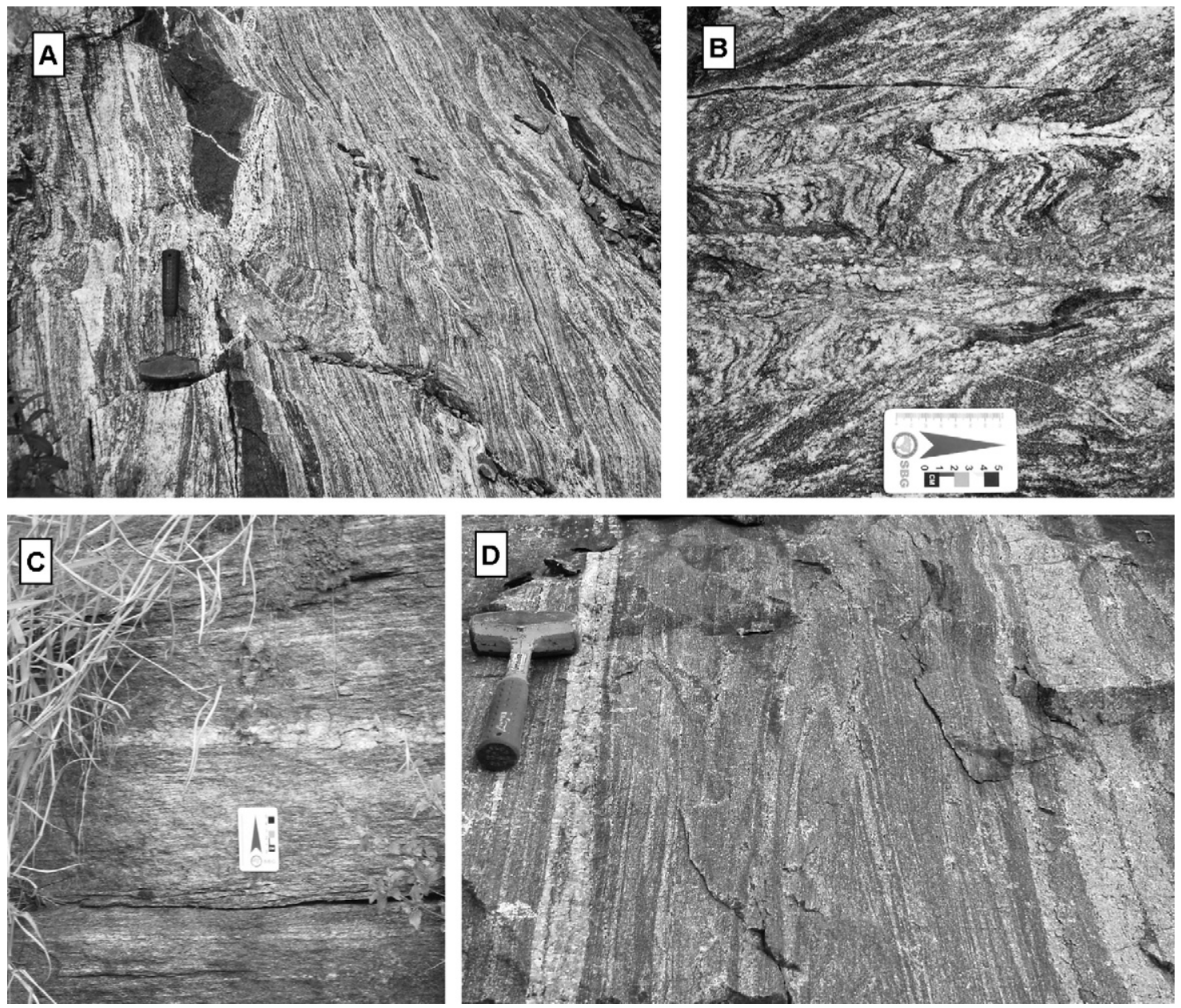

Fig. 3. (A) Banded tonalite gneiss with disrupted mafic bodies and leucossome veins (Mantiqueira Complex, sample RP-1); (B) in the same outcrop as previous picture, the gneiss displays a former banded structure partially transposed by the flat-lying regional Brasiliano foliation; (C) homogeneous granodiorite gneiss strongly foliated (Mantiqueira Complex, sample LC-67); (D) Enderbite gneiss with light leucossome bands of charnockitic composition (Juiz de For a Complex, sample Ub-1).

Granulite facies orthogneisses of the Juiz de Fora Complex are often migmatized. One investigated outcrop consists of a complex folded, stromatic migmatite with pink granite veins that display concordant to crosscutting relations with the migmatitic banding. Sample LC-66a is a mafic granulite, composed of plagioclase and pyroxenes, taken from a dark-green paleosome band. Pyroxenes can be replaced by hornblende, by its turn replaced by minor biotite. Quartz and opaque minerals are the main accessory minerals. Chemical composition suggests a gabbro-diorite protolith for this rock (Table 1).

Sample Ub-1 is a migmatized enderbite gneiss rich in leucosome veins of light green charnockite (Fig. 3d), and also with mafic enclaves (basic granulite). The enderbite is composed of plagioclase, quartz, orthopyroxene and clinopyroxene, with biotite and hornblende as retrograde minerals. Leucosome veins are generally less than one to a few centimeters wide, and parallel to the gneiss foliation. It was not possible to separate them from the collected sample.

\section{Isotopic results}

\subsection{Analytical procedures}

Zircon, titanite and monazite concentrates were extracted from rock samples using conventional gravimetric and magnetic (Frantz isodynamic separator) techniques. Final separation was achieved by hand picking using a binocular microscope. Zircon SHRIMP analyses were carried out at the Research School of Earth Sciences, Australian National University. Zircon grains were mounted in a 1 in. diameter epoxy disk with standard RSES zircon crystals and sectioned approxi- 
Table 2

Summary of SHRIMP U-Pb zircon data for Mantiqueira gneiss samples

\begin{tabular}{|c|c|c|c|c|c|c|c|c|c|c|c|c|c|c|c|}
\hline $\begin{array}{l}\text { Grain } \\
\text { spot }\end{array}$ & $\%^{206} \mathrm{Pbc}$ & $\mathrm{U}(\mathrm{ppm})$ & $\begin{array}{l}\text { Th } \\
(\mathrm{ppm})\end{array}$ & $\begin{array}{l}{ }^{232} \mathrm{Th} / \\
{ }^{238} \mathrm{U}\end{array}$ & $\begin{array}{l}{ }^{206} \mathrm{~Pb}^{\mathrm{a}} \\
(\mathrm{ppm})\end{array}$ & $\begin{array}{l}{ }^{206} \mathrm{~Pb} /{ }^{238} \mathrm{U} \\
\text { age }^{\mathrm{b}}\end{array}$ & $\begin{array}{l}{ }^{207} \mathrm{~Pb} / \\
{ }^{206} \mathrm{~Pb} \text { age }\end{array}$ & $\%$ discordant & $\begin{array}{l}{ }^{207} \mathrm{~Pb}^{\mathrm{a}} / \\
{ }^{206} \mathrm{~Pb}^{\mathrm{a}, \mathrm{b}}\end{array}$ & $\pm \%$ & $\begin{array}{l}{ }^{207} \mathrm{~Pb}^{\mathrm{a}} / \\
{ }^{235} \mathrm{U}^{\mathrm{b}}\end{array}$ & $\pm \%$ & $\begin{array}{l}{ }^{206} \mathrm{~Pb}^{\mathrm{a} /} \\
{ }^{238} \mathrm{U}^{\mathrm{b}}\end{array}$ & $\pm \%$ & $\begin{array}{l}\text { Error } \\
\text { correction }\end{array}$ \\
\hline \multicolumn{16}{|c|}{ Sample RP-01 } \\
\hline 1.1 & 0.02 & 158 & 52 & 0.34 & & $2,656 \pm 25$ & $3096.8 \pm 4.5$ & 14 & 0.23653 & 0.28 & 16.62 & 1.2 & 0.5097 & 1.1 & 0.971 \\
\hline 1.2 & 0.04 & 320 & 121 & 0.39 & 126.0 & $2,425 \pm 24$ & $3148.8 \pm 4.2$ & 23 & 0.24439 & 0.26 & 15.39 & 1.2 & 0.4568 & 1.2 & 0.976 \\
\hline 2.1 & 0.02 & 257 & 171 & 0.69 & 116.0 & $2,723 \pm 25$ & $2876.3 \pm 3.6$ & 5 & 0.20625 & 0.22 & 14.95 & 1.1 & 0.5257 & 1.1 & 0.981 \\
\hline 2.2 & 0.03 & 366 & 50 & 0.14 & 114.0 & $1,990 \pm 19$ & $2932.7 \pm 6.7$ & 32 & 0.21356 & 0.42 & 10.65 & 1.2 & 0.3616 & 1.1 & 0.937 \\
\hline 3.1 & 0.03 & 62 & 52 & 0.87 & 34.2 & $3,182 \pm 36$ & $3224.2 \pm 5.9$ & 1 & 0.25631 & 0.37 & 22.56 & 1.5 & 0.6382 & 1.4 & 0.967 \\
\hline 4.1 & 0.02 & 99 & 63 & 0.65 & 30.5 & $1,974 \pm 20$ & $2110.1 \pm 8.6$ & 6 & 0.1309 & 0.49 & 6,467 & 1.3 & 0.3583 & 1.2 & 0.926 \\
\hline 5.1 & 0.01 & 271 & 85 & 0.33 & 116.0 & $2,608 \pm 29$ & $2828.0 \pm 3.6$ & 8 & 0.20022 & 0.22 & 13.77 & 1.4 & 0.4987 & 1.3 & 0.987 \\
\hline 6.1 & 0.02 & 279 & 96 & 0.36 & 86.2 & $1,982 \pm 22$ & $2635.7 \pm 7.6$ & 25 & 0.17815 & 0.46 & 8.84 & 1.4 & 0.36 & 1.3 & 0.941 \\
\hline 6.2 & 0.01 & 240 & 97 & 0.42 & 123.0 & $3,014 \pm 27$ & $3232.3 \pm 4.4$ & 7 & 0.25763 & 0.28 & 21.17 & 1.2 & 0.5961 & 1.1 & 0.971 \\
\hline 7.1 & 0.10 & 113 & 91 & 0.84 & 36.8 & $2,079 \pm 22$ & $2131.8 \pm 8.8$ & 2 & 0.13253 & 0.5 & 6,955 & 1.3 & 0.3806 & 1.2 & 0.923 \\
\hline 8.1 & 0.02 & 208 & 100 & 0.50 & 102.0 & $2,922 \pm 27$ & $3229.2 \pm 3.4$ & 10 & 0.25712 & 0.22 & 20.33 & 1.2 & 0.5734 & 1.1 & 0.982 \\
\hline 8.2 & 0.02 & 397 & 82 & 0.21 & 120.0 & $1,950 \pm 20$ & $2671.2 \pm 5.4$ & 27 & 0.182 & 0.33 & 8.86 & 1.2 & 0.3532 & 1.2 & 0.963 \\
\hline 9.1 & 0.04 & 124 & 109 & 0.91 & 62.1 & $2,970 \pm 33$ & $3168.3 \pm 4.5$ & 6 & 0.24741 & 0.28 & 19.96 & 1.4 & 0.5851 & 1.4 & 0.980 \\
\hline 9.2 & 0.08 & 342 & 226 & 0.68 & 88.5 & $1,696 \pm 18$ & $2886.5 \pm 4.3$ & 41 & 0.20755 & 0.27 & 8.61 & 1.2 & 0.3009 & 1.2 & 0.976 \\
\hline 10.1 & 0.00 & 322 & 210 & 0.67 & 154.0 & $2,854 \pm 26$ & $3263.4 \pm 2.9$ & 13 & 0.26277 & 0.18 & 20.18 & 1.1 & 0.5569 & 1.1 & 0.987 \\
\hline 10.2 & 0.07 & 127 & 53 & 0.44 & 45.6 & $2,253 \pm 23$ & $2403.4 \pm 6.7$ & 6 & 0.15514 & 0.4 & 8.95 & 1.3 & 0.4184 & 1.2 & 0.952 \\
\hline 11.1 & 0.01 & 134 & 106 & 0.82 & 61.5 & $2,756 \pm 26$ & $2982.8 \pm 8.3$ & 8 & 0.2203 & 0.52 & 16.2 & 1.3 & 0.5334 & 1.2 & 0.914 \\
\hline 11.2 & 0.06 & 91 & 66 & 0.74 & 26.6 & $1,880 \pm 20$ & $2126 \pm 10$ & 12 & 0.13213 & 0.59 & 6,169 & 1.4 & 0.3386 & 1.2 & 0.902 \\
\hline 12.1 & 0.04 & 98 & 36 & 0.38 & 36.4 & $2,319 \pm 23$ & $2738.2 \pm 8.2$ & 15 & 0.18955 & 0.5 & 11.32 & 1.3 & 0.433 & 1.2 & 0.923 \\
\hline 12.2 & 0.00 & 403 & 114 & 0.29 & 112.0 & $1,804 \pm 18$ & $2095.7 \pm 4.5$ & 14 & 0.12984 & 0.26 & 5,782 & 1.1 & 0.323 & 1.1 & 0.974 \\
\hline 13.1 & 0.00 & 169 & 74 & 0.45 & 79.0 & $2,803 \pm 26$ & $2975.4 \pm 4.2$ & 6 & 0.21928 & 0.26 & 16.46 & 1.2 & 0.5446 & 1.2 & 0.976 \\
\hline 14.1 & 0.00 & 331 & 90 & 0.28 & 98.4 & $1,915 \pm 19$ & $3083 \pm 11$ & 38 & 0.2345 & 0.72 & 11.19 & 1.3 & 0.346 & 1.1 & 0.845 \\
\hline 14.2 & 0.02 & 94 & 53 & 0.59 & 28.1 & $1,929 \pm 20$ & $2117.1 \pm 9.4$ & 9 & 0.13143 & 0.53 & 6,321 & 1.3 & 0.3488 & 1.2 & 0.915 \\
\hline 15.1 & 0.01 & 272 & 121 & 0.46 & 120.0 & $2,665 \pm 25$ & $3218.1 \pm 4.2$ & 17 & 0.25532 & 0.27 & 18.02 & 1.2 & 0.512 & 1.1 & 0.974 \\
\hline 16.1 & 0.04 & 441 & 58 & 0.14 & 119.0 & $1,767 \pm 18$ & $2675 \pm 11$ & 34 & 0.1825 & 0.63 & 7.93 & 1.3 & 0.3154 & 1.2 & 0.880 \\
\hline 16.2 & 0.01 & 141 & 80 & 0.58 & 41.4 & $1,897 \pm 19$ & $2521.4 \pm 6.8$ & 25 & 0.16636 & 0.4 & 7,846 & 1.2 & 0.3421 & 1.2 & 0.946 \\
\hline \multicolumn{16}{|c|}{ Sample LC-70 } \\
\hline 1.1 & 0.01 & 449 & 304 & 0.70 & 197.0 & $2,658 \pm 16$ & $2688.6 \pm 3.8$ & 1 & 0.18393 & 0.23 & 12.94 & 0.8 & 0.5102 & 0.8 & 0.958 \\
\hline 1.2 & 0.01 & 885 & 171 & 0.20 & 276.0 & $1,999 \pm 12$ & $2077.6 \pm 3.8$ & 4 & 0.1285 & 0.22 & 6,441 & 0.8 & 0.3635 & 0.7 & 0.958 \\
\hline 2.1 & 0.04 & 121 & 32 & 0.27 & 49.3 & $2,512 \pm 19$ & $2616.3 \pm 8.5$ & 4 & 0.17608 & 0.51 & 11.57 & 1 & 0.4764 & 0.9 & 0.870 \\
\hline 3.1 & 0.01 & 1296 & 431 & 0.34 & 409.0 & $2,016 \pm 12$ & $2042.6 \pm 3.4$ & 1 & 0.12598 & 0.19 & 6,377 & 0.7 & 0.3671 & 0.7 & 0.965 \\
\hline 3.2 & 0.03 & 282 & 129 & 0.47 & 123.0 & $2,640 \pm 17$ & $2674.7 \pm 4.7$ & 1 & 0.18238 & 0.28 & 12.73 & 0.8 & 0.5061 & 0.8 & 0.941 \\
\hline 4.1 & 0.00 & 683 & 264 & 0.40 & 213.0 & $1,995 \pm 13$ & $2045.0 \pm 4.3$ & 2 & 0.12615 & 0.24 & 6,309 & 0.8 & 0.3627 & 0.7 & 0.950 \\
\hline 5.1 & 0.08 & 207 & 10 & 0.05 & 84.8 & $2,508 \pm 23$ & $2600 \pm 11$ & 4 & 0.1743 & 0.68 & 11.43 & 1.3 & 0.4755 & 1.1 & 0.849 \\
\hline 6.1 & 0.03 & 952 & 454 & 0.49 & 337.0 & $2,224 \pm 13$ & $2512.8 \pm 5.9$ & 11 & 0.16552 & 0.35 & 9,404 & 0.8 & 0.4121 & 0.7 & 0.898 \\
\hline 7.1 & 0.13 & 866 & 168 & 0.20 & 269.0 & $1,986 \pm 12$ & $2107.6 \pm 4.6$ & 6 & 0.13072 & 0.26 & 6,504 & 0.8 & 0.3609 & 0.7 & 0.940 \\
\hline 8.1 & 0.08 & 310 & 174 & 0.58 & 114.0 & $2,297 \pm 18$ & $2543.0 \pm 5.1$ & 10 & 0.16852 & 0.3 & 9,947 & 10 & 0.4281 & 1 & 0.952 \\
\hline 9.1 & 0.32 & 119 & 95 & 0.82 & 48.2 & $2,483 \pm 18$ & $2681.2 \pm 8.6$ & 7 & 0.18311 & 0.52 & 11.87 & 1 & 0.47 & 0.9 & 0.859 \\
\hline 10.1 & 0.05 & 350 & 229 & 0.67 & 151.0 & $2,625 \pm 16$ & $2691.4 \pm 4.1$ & 2 & 0.18424 & 0.25 & 12.77 & 0.8 & 0.5027 & 0.8 & 0.951 \\
\hline 10.2 & 0.04 & 304 & 114 & 0.39 & 124.0 & $2,499 \pm 16$ & $2582.4 \pm 7.7$ & 3 & 0.17254 & 0.46 & 11.26 & 0.9 & 0.4735 & 0.8 & 0.858 \\
\hline 11.1 & 0.01 & 1674 & 25 & 0.02 & 536.0 & $2,041 \pm 12$ & $2043.6 \pm 2.9$ & 0 & 0.12605 & 0.17 & 6,474 & 0.7 & 0.3725 & 0.7 & 0.974 \\
\hline 12.1 & 0.00 & 986 & 416 & 0.44 & 297.0 & $1,938 \pm 12$ & $2037.7 \pm 3.6$ & 5 & 0.12563 & 0.2 & 6,073 & 0.8 & 0.3506 & 0.7 & 0.962 \\
\hline 13.1 & 0.09 & 120 & 66 & 0.57 & 51.9 & $2,618 \pm 20$ & $2680.1 \pm 6.9$ & 2 & 0.18298 & 0.42 & 12.64 & 1 & 0.501 & 0.9 & 0.911 \\
\hline 14.1 & 0.04 & 324 & 231 & 0.74 & 126.0 & $2,409 \pm 15$ & $2528.2 \pm 4.7$ & 5 & 0.16704 & 0.28 & 10,438 & 0.8 & 0.4532 & 0.8 & 0.940 \\
\hline 15.1 & 0.01 & 723 & 324 & 0.46 & 219.0 & $1,948 \pm 12$ & $2023.7 \pm 4.5$ & 4 & 0.12464 & 0.25 & 6,064 & 0.8 & 0.3529 & 0.7 & 0.945 \\
\hline \multicolumn{16}{|c|}{ Sample LC-77 } \\
\hline 1.1 & 0.00 & 1916 & 34 & 0.02 & 599.0 & $2,002 \pm 16$ & $2018.3 \pm 3.1$ & & 0.12426 & 0.17 & 6,239 & 0.9 & 0.3641 & 0.9 & 0.983 \\
\hline 1.2 & 0.02 & 56 & 38 & 0.71 & 25.7 & $2,777 \pm 29$ & $2727 \pm 18$ & -2 & 0.1882 & 1.1 & 13.98 & 1.7 & 0.5385 & 1.3 & 0.770 \\
\hline 2.1 & 0.04 & 813 & 14 & 0.02 & 265.0 & $2,072 \pm 17$ & $2067.8 \pm 4.4$ & & 0.12779 & 0.25 & 6,680 & 1 & 0.3791 & 1 & 0.967 \\
\hline 2.2 & 0.26 & 64 & 30 & 0.48 & 24.2 & $2,358 \pm 25$ & $2598 \pm 23$ & 9 & 0.1742 & 1.4 & 10.61 & 1.9 & 0.4416 & 1.3 & 0.675 \\
\hline 3.1 & 0.00 & 155 & 82 & 0.55 & 70.6 & $2,748 \pm 24$ & $2696 \pm 18$ & -2 & 0.1847 & 1.1 & 13.54 & 1.5 & 0.5316 & 1.1 & 0.694 \\
\hline 3.2 & 0.00 & 2426 & 53 & 0.02 & 799.0 & $2,092 \pm 17$ & $2039.0 \pm 4.9$ & & 0.12572 & 0.28 & 6,647 & 1 & 0.3834 & 0.9 & 0.958 \\
\hline 4.1 & 0.00 & 44 & 26 & 0.61 & 19.5 & $2,659 \pm 30$ & $2693 \pm 14$ & 1 & 0.1844 & 0.85 & 12.98 & 1.6 & 0.5105 & 1.4 & 0.849 \\
\hline 4.2 & 0.45 & 1624 & 30 & 0.02 & 463.0 & $1,841 \pm 15$ & $2050.5 \pm 9.1$ & & 0.12655 & 0.52 & 5,767 & 1.1 & 0.3305 & 0.9 & 0.875 \\
\hline 5.1 & 0.24 & 1763 & 216 & 0.13 & 542.0 & $1,969 \pm 16$ & $2311.7 \pm 6.7$ & & 0.14704 & 0.39 & 7,241 & 1 & 0.3572 & 0.9 & 0.922 \\
\hline 5.2 & 0.03 & 840 & 498 & 0.61 & 462.0 & $3,190 \pm 24$ & $3145.2 \pm 2.7$ & & 0.24384 & 0.17 & 21.52 & 1 & 0.6402 & 1 & 0.984 \\
\hline 6.1 & -0.01 & 154 & 100 & 0.67 & 76.8 & $2,948 \pm 25$ & $2935.1 \pm 5.9$ & & 0.21386 & 0.37 & 17.1 & 1.1 & 0.5798 & 1.1 & 0.946 \\
\hline 7.1 & 0.06 & 45 & 28 & 0.64 & 20.6 & $2,746 \pm 39$ & $2728 \pm 19$ & -1 & 0.1884 & 1.1 & 13.8 & 2.1 & 0.531 & 1.8 & 0.838 \\
\hline 8.1 & 0.06 & 436 & 131 & 0.31 & 156.0 & $2,243 \pm 18$ & $2551.7 \pm 6.8$ & & 0.1694 & 0.4 & 9.72 & 1 & 0.4161 & 1 & 0.922 \\
\hline 9.1 & 0.12 & 967 & 196 & 0.21 & 328.0 & $2,142 \pm 17$ & $2328.0 \pm 4.8$ & & 0.14844 & 0.28 & 8,065 & 1 & 0.394 & 0.9 & 0.959 \\
\hline 10.1 & 0.02 & 187 & 92 & 0.51 & 85.6 & $2,749 \pm 23$ & $2721.2 \pm 5.8$ & & 0.18759 & 0.35 & 13.76 & 1.1 & 0.5319 & 1 & 0.947 \\
\hline 11.1 & 0.02 & 384 & 54 & 0.15 & 196.0 & $3,005 \pm 24$ & $2960.3 \pm 7.1$ & & 0.21723 & 0.44 & 17.79 & 1.1 & 0.5939 & 1 & 0.915 \\
\hline 12.1 & 0.01 & 357 & 182 & 0.53 & 170.0 & $2,838 \pm 25$ & $2912.5 \pm 3.7$ & & 0.21091 & 0.23 & 16.08 & 1.1 & 0.553 & 1.1 & 0.978 \\
\hline 12.2 & 0.13 & 1065 & 167 & 0.16 & 317.0 & $1,915 \pm 18$ & $2383.2 \pm 7.9$ & & 0.15332 & 0.46 & 7,314 & 1.2 & 0.346 & 1.1 & 0.918 \\
\hline 13.1 & 0.00 & 436 & 177 & 0.42 & 190.0 & $2,644 \pm 21$ & $2684.2 \pm 5.7$ & & 0.18344 & 0.35 & 12.82 & 1 & 0.507 & 1 & 0.941 \\
\hline 14.1 & 0.04 & 81 & 48 & 0.62 & 35.3 & $2,657 \pm 25$ & $2730.9 \pm 8.3$ & & 0.18871 & 0.5 & 13.27 & 1.2 & 0.5102 & 1.1 & 0.915 \\
\hline \multicolumn{16}{|c|}{ Sample LC-79 } \\
\hline 1.1 & 0.03 & 205 & 115 & 0.58 & 98.8 & $2,866 \pm 21$ & $2885.8 \pm 7.7$ & 1 & 0.20745 & 0.47 & 16.01 & 1 & 0.5597 & 0.9 & 0.886 \\
\hline 2.1 & 0.01 & 1273 & 129 & 0.10 & 596.0 & $2,803 \pm 16$ & $2827.5 \pm 2.4$ & 1 & 0.20015 & 0.15 & 15.03 & 0.7 & 0.5446 & 0.7 & 0.979 \\
\hline 3.1 & 0.01 & 855 & 23 & 0.03 & 356.0 & $2,547 \pm 15$ & $2734.1 \pm 3.0$ & 7 & 0.18908 & 0.18 & 12,633 & 0.8 & 0.4846 & 0.7 & 0.969 \\
\hline
\end{tabular}


Table 2 (Continued)

\begin{tabular}{|c|c|c|c|c|c|c|c|c|c|c|c|c|c|c|c|}
\hline $\begin{array}{l}\text { Grain } \\
\text { spot }\end{array}$ & $\%^{206} \mathrm{Pbc}$ & $\mathrm{U}(\mathrm{ppm})$ & $\begin{array}{l}\text { Th } \\
(\mathrm{ppm})\end{array}$ & $\begin{array}{l}{ }^{232} \mathrm{Th} / \\
{ }^{238} \mathrm{U}\end{array}$ & $\begin{array}{l}{ }^{206} \mathrm{~Pb}^{\mathrm{a}} \\
(\mathrm{ppm})\end{array}$ & $\begin{array}{l}{ }^{206} \mathrm{~Pb} /{ }^{238} \mathrm{U} \\
\mathrm{age}^{\mathrm{b}}\end{array}$ & $\begin{array}{l}{ }^{207} \mathrm{~Pb} / \\
{ }^{206} \mathrm{~Pb} \text { age }\end{array}$ & $\%$ discordant & $\begin{array}{l}{ }^{207} \mathrm{~Pb}^{\mathrm{a}} / \\
{ }^{206} \mathrm{~Pb}^{\mathrm{a}, \mathrm{b}}\end{array}$ & $\pm \%$ & $\begin{array}{l}{ }^{207} \mathrm{~Pb}^{\mathrm{a}} / \\
{ }^{235} \mathrm{U}^{\mathrm{b}}\end{array}$ & $\pm \%$ & $\begin{array}{l}{ }^{206} \mathrm{~Pb}^{\mathrm{a}} / \\
{ }^{238} \mathrm{U}^{\mathrm{b}}\end{array}$ & $\pm \%$ & $\begin{array}{l}\text { Error } \\
\text { correction }\end{array}$ \\
\hline$\overline{4.1}$ & 0.07 & 1016 & 346 & 0.35 & 284.0 & $1,812 \pm 11$ & $2022.2 \pm 4.1$ & 10 & 0.12453 & 0.23 & 5,573 & 0.8 & 0.3246 & 0.7 & 0.952 \\
\hline 4.2 & 0.25 & 3935 & 904 & 0.24 & 546.0 & $963.4 \pm 6.3$ & $1603.4 \pm 8.3$ & 40 & 0.0989 & 0.44 & 2,198 & 0.8 & 0.1612 & 0.7 & 0.847 \\
\hline 5.1 & 0.11 & 3216 & 765 & 0.25 & 619.0 & $1,302 \pm 10$ & $1823.1 \pm 3.8$ & 29 & 0.11145 & 0.21 & 3,440 & 0.9 & 0.2238 & 0.9 & 0.972 \\
\hline 6.1 & 0.67 & 2881 & 108 & 0.04 & 432.0 & $1031.0 \pm 7.7$ & $1633 \pm 2$ & 37 & 0.1005 & 1.2 & 2,403 & 1.5 & 0.1734 & 0.8 & 0.555 \\
\hline 7.1 & 0.01 & 1367 & 555 & 0.42 & 414.0 & $1,947 \pm 12$ & $2029.3 \pm 4.8$ & 4 & 0.12503 & 0.27 & 6,080 & 0.8 & 0.3527 & 0.7 & 0.934 \\
\hline 8.1 & 0.02 & 2064 & 478 & 0.24 & 481.0 & $1545.9 \pm 9.7$ & $1923.0 \pm 3.7$ & 20 & 0.11779 & 0.2 & 4,401 & 0.7 & 0.271 & 0.7 & 0.961 \\
\hline 9.1 & 0.07 & 1166 & 266 & 0.24 & 348.0 & $1923 \pm 12$ & $2034.5 \pm 8.5$ & 5 & 0.1254 & 0.48 & 6,010 & 0.9 & 0.3476 & 0.7 & 0.838 \\
\hline 10.1 & 0.05 & 492 & 33 & 0.07 & 198.0 & $2,479 \pm 15$ & $2759.7 \pm 5.1$ & 10 & 0.19204 & 0.31 & 12.42 & 0.8 & 0.469 & 0.8 & 0.923 \\
\hline 11.1 & 0.11 & 926 & 328 & 0.37 & 282.0 & $1,955 \pm 12$ & $2043.2 \pm 4.4$ & 4 & 0.12602 & 0.25 & 6,155 & 0.8 & 0.3542 & 0.7 & 0.947 \\
\hline 11.2 & 0.01 & 1585 & 768 & 0.50 & 470.0 & $1,911 \pm 13$ & $2010.4 \pm 5.4$ & 5 & 0.12371 & 0.31 & 5,885 & 0.8 & 0.345 & 0.8 & 0.928 \\
\hline 12.1 & 0.07 & 185 & 105 & 0.59 & 58.1 & $2,004 \pm 22$ & $2044 \pm 0$ & 2 & 0.1261 & 1.1 & 6.34 & 1.7 & 0.3646 & 1.3 & 0.739 \\
\hline 13.1 & 0.00 & 1318 & 34 & 0.03 & 555.0 & $2,572 \pm 15$ & $2728.3 \pm 4.1$ & 6 & 0.18841 & 0.25 & 12,738 & 0.8 & 0.4904 & 0.7 & 0.945 \\
\hline 14.1 & 0.03 & 1691 & 569 & 0.35 & 411.0 & $1,607 \pm 10$ & $1933.5 \pm 3.6$ & 17 & 0.11849 & 0.2 & 4,625 & 0.8 & 0.2831 & 0.7 & 0.963 \\
\hline 15.1 & 0.01 & 651 & 486 & 0.77 & 282.0 & $2,636 \pm 16$ & $2708.8 \pm 5.1$ & 3 & 0.18619 & 0.31 & 12.97 & 0.8 & 0.5052 & 0.7 & 0.923 \\
\hline 16.1 & 0.00 & 1348 & 489 & 0.37 & 417.0 & $1,983 \pm 19$ & $2030.6 \pm 9.3$ & 2 & 0.12513 & 0.52 & 6,215 & 1.2 & 0.3602 & 1.1 & 0.903 \\
\hline \multicolumn{16}{|c|}{ Sample LC-78 } \\
\hline 1.1 & 0.00 & 186 & 103 & 0.57 & 49.8 & $1,748 \pm 20$ & $1972.7 \pm 9.8$ & 11 & 0.12111 & 0.61 & 5,201 & 1.4 & 0.3115 & 1.3 & 0.920 \\
\hline 2.1 & 0.01 & 1608 & 538 & 0.35 & 537.0 & $2,116 \pm 22$ & $2597 \pm 10$ & 19 & 0.174 & 0.42 & 9.32 & 1.4 & 0.3885 & 1.2 & 0.896 \\
\hline 3.1 & 0.00 & 316 & 156 & 0.51 & 92.2 & $1,883 \pm 20$ & $2022.8 \pm 7.4$ & 7 & 0.12458 & 0.66 & 5,828 & 1.3 & 0.3393 & 1.3 & 0.948 \\
\hline 4.1 & 0.00 & 214 & 217 & 1.05 & 65.5 & $1,968 \pm 22$ & $2029 \pm 12$ & 3 & 0.12504 & 0.8 & 6,157 & 1.4 & 0.3571 & 1.3 & 0.887 \\
\hline 5.1 & 0.04 & 288 & 129 & 0.46 & 88.5 & $1,968 \pm 23$ & $2061 \pm 14$ & 5 & 0.1273 & 0.95 & 6,264 & 1.6 & 0.357 & 1.3 & 0.859 \\
\hline 6.1 & 0.10 & 230 & 180 & 0.81 & 57.6 & $1,645 \pm 22$ & $1923 \pm 17$ & 14 & 0.1178 & 0.4 & 4,722 & 1.8 & 0.2908 & 1.5 & 0.844 \\
\hline 7.1 & 0.07 & 328 & 158 & 0.50 & 97.3 & $1,914 \pm 21$ & $2040.1 \pm 7.0$ & 6 & 0.1258 & 0.31 & 5,995 & 1.3 & 0.3456 & 1.2 & 0.953 \\
\hline 8.1 & 0.00 & 431 & 509 & 1.22 & 141.0 & $2,082 \pm 22$ & $2048.8 \pm 5.5$ & -2 & 0.12642 & 0.35 & 6,644 & 1.3 & 0.3811 & 1.2 & 0.970 \\
\hline 8.2 & 0.03 & 373 & 452 & 1.25 & 113.0 & $1,943 \pm 21$ & $2034.7 \pm 6.1$ & 5 & 0.12542 & 0.74 & 6,083 & 1.3 & 0.3518 & 1.2 & 0.963 \\
\hline 9.1 & 0.00 & 249 & 187 & 0.78 & 96.8 & $2,407 \pm 26$ & $2693 \pm 12$ & 11 & 0.1844 & 0.39 & 11.51 & 1.5 & 0.4526 & 1.3 & 0.872 \\
\hline 10.1 & 0.00 & 604 & 479 & 0.82 & 280.0 & $2,779 \pm 28$ & $2728.8 \pm 6.5$ & -2 & 0.18846 & 1.9 & 14 & 1.3 & 0.5388 & 1.2 & 0.952 \\
\hline 10.2 & 0.00 & 191 & 141 & 0.76 & 57.8 & $1,944 \pm 22$ & $2041 \pm 34$ & 5 & 0.1259 & 0.32 & 6.11 & 2.3 & 0.3519 & 1.3 & 0.562 \\
\hline 7.2 & -0.02 & 226 & 182 & 0.83 & 96.8 & $2,612 \pm 27$ & $2673.1 \pm 5.4$ & 2 & 0.18221 & 0.44 & 12.55 & 1.3 & 0.4996 & 1.3 & 0.969 \\
\hline 11.1 & -0.01 & 586 & 252 & 0.45 & 243.0 & $2,543 \pm 26$ & $2652.2 \pm 7.2$ & 4 & 0.17992 & 0.52 & 12 & 1.3 & 0.4836 & 1.2 & 0.941 \\
\hline 12.1 & 0.03 & 150 & 147 & 1.01 & 42.7 & $1,850 \pm 23$ & $2026.8 \pm 9.3$ & 9 & 0.12486 & 0.53 & 5,721 & 1.5 & 0.3323 & 1.4 & 0.937 \\
\hline 13.1 & 0.07 & 161 & 136 & 0.87 & 51.5 & $2,041 \pm 23$ & $2027.3 \pm 9.5$ & -1 & 0.12489 & 0.46 & 6,414 & 1.4 & 0.3725 & 1.3 & 0.925 \\
\hline 13.2 & 0.00 & 226 & 155 & 0.71 & 66.5 & $1,901 \pm 21$ & $2020.1 \pm 8.1$ & 6 & 0.12439 & 2.7 & 5,883 & 1.4 & 0.343 & 1.3 & 0.941 \\
\hline 13.3 & 0.81 & 25 & 9 & 0.37 & 3.7 & $1,012 \pm 17$ & $1538 \pm 50$ & 34 & 0.0955 & 0.61 & 2,238 & 3.2 & 0.1699 & 1.8 & 0.565 \\
\hline \multicolumn{16}{|c|}{ Sample LC-67 } \\
\hline 1.1 & 0.02 & 192 & 38 & 0.21 & 84.1 & $2,650 \pm 36$ & $2697.0 \pm 5.2$ & 2 & 0.18486 & 0.31 & 12.96 & 1.7 & 0.5085 & 1.7 & 0.982 \\
\hline 2.1 & 0.02 & 311 & 138 & 0.46 & 173.0 & $3,225 \pm 33$ & $3219.8 \pm 3.2$ & 0 & 0.2556 & 0.2 & 22.88 & 1.3 & 0.6492 & 1.3 & 0.988 \\
\hline 3.1 & 0.04 & 126 & 86 & 0.71 & 40.3 & $2,043 \pm 23$ & $2053.3 \pm 9.8$ & 1 & 0.12675 & 0.56 & 6,516 & 1.4 & 0.3729 & 1.3 & 0.922 \\
\hline 3.2 & 0.03 & 165 & 88 & 0.55 & 88.1 & $3,123 \pm 32$ & $3188.1 \pm 5.3$ & 2 & 0.25052 & 0.34 & 21.53 & 1.3 & 0.6232 & 1.3 & 0.968 \\
\hline 4.1 & 0.00 & 320 & 117 & 0.38 & 91.9 & $1,859 \pm 21$ & $2031.3 \pm 6.2$ & 8 & 0.12518 & 0.35 & 5,768 & 1.4 & 0.3342 & 1.3 & 0.966 \\
\hline 5.1 & 0.00 & 493 & 196 & 0.41 & 222.0 & $2,715 \pm 27$ & $3203.2 \pm 5.8$ & 15 & 0.25293 & 0.37 & 18.27 & 1.3 & 0.5238 & 1.2 & 0.958 \\
\hline 6.1 & 0.00 & 105 & 70 & 0.69 & 32.4 & $1,985 \pm 23$ & $2040.8 \pm 10.0$ & 3 & 0.12585 & 0.56 & 6,257 & 1.5 & 0.3606 & 1.3 & 0.922 \\
\hline 6.2 & 0.00 & 531 & 36 & 0.07 & 150.0 & $1,835 \pm 20$ & $2015.6 \pm 4.9$ & 9 & 0.12407 & 0.28 & 5,634 & 1.3 & 0.3293 & 1.2 & 0.976 \\
\hline 7.1 & 0.10 & 102 & 77 & 0.79 & 31.5 & $1,984 \pm 23$ & $2050 \pm 11$ & 3 & 0.12652 & 0.6 & 6,288 & 1.5 & 0.3604 & 1.3 & 0.912 \\
\hline 8.1 & 1.25 & 164 & 10 & 0.07 & 53.9 & $2,066 \pm 24$ & $2393 \pm 54$ & 14 & 0.1542 & 3.2 & 8.03 & 3.5 & 0.3778 & 1.4 & 0.399 \\
\hline 8.2 & 0.00 & 268 & 139 & 0.53 & 82.4 & $1,970 \pm 21$ & $2029.9 \pm 6.0$ & 3 & 0.12508 & 0.34 & 6,163 & 1.3 & 0.3574 & 1.3 & 0.965 \\
\hline 9.1 & -0.01 & 297 & 115 & 0.40 & 118.0 & $2,457 \pm 26$ & $2833 \pm 13$ & 13 & 0.2008 & 0.78 & 12.84 & 1.5 & 0.4638 & 1.3 & 0.857 \\
\hline 10.1 & 0.02 & 109 & 52 & 0.50 & 46.0 & $2,568 \pm 28$ & $2898 \pm 11$ & 1 & 0.2091 & 0.65 & 14.11 & 1.5 & 0.4894 & 1.3 & 0.898 \\
\hline 11.1 & 0.01 & 128 & 79 & 0.64 & 67.0 & $3,073 \pm 34$ & $3127.1 \pm 6.3$ & 2 & 0.24107 & 0.39 & 20.3 & 1.5 & 0.6108 & 1.4 & 0.963 \\
\hline 11.2 & 0.04 & 96 & 55 & 0.59 & 31.1 & $2,054 \pm 24$ & $2053 \pm 10$ & 0 & 0.12675 & 0.57 & 6,558 & 1.5 & 0.3752 & 1.3 & 0.919 \\
\hline 12.1 & 0.01 & 147 & 106 & 0.75 & 54.3 & $2,309 \pm 25$ & $2528 \pm 18$ & 9 & 0.167 & 1.1 & 9.92 & 1.7 & 0.4308 & 1.3 & 0.767 \\
\hline 12.2 & 0.03 & 178 & 69 & 0.40 & 83.5 & $2,812 \pm 29$ & $3141 \pm 22$ & 10 & 0.2432 & 1.4 & 18.34 & 1.9 & 0.5469 & 1.3 & 0.682 \\
\hline 13.1 & 0.00 & 74 & 118 & 1.64 & 22.1 & $1,916 \pm 26$ & $2047 \pm 12$ & 6 & 0.12633 & 0.69 & 6.03 & 1.7 & 0.346 & 1.6 & 0.916 \\
\hline 14.1 & 0.00 & 542 & 200 & 0.38 & 237.0 & $2,650 \pm 27$ & $3045 \pm 12$ & 13 & 0.229 & 0.77 & 16.06 & 1.5 & 0.5085 & 1.2 & 0.847 \\
\hline 14.2 & 0.00 & 221 & 87 & 0.41 & 63.8 & $1,870 \pm 21$ & $2046.4 \pm 7.4$ & 9 & 0.12625 & 0.42 & 5,858 & 1.4 & 0.3365 & 1.3 & 0.951 \\
\hline 15.1 & -0.01 & 155 & 55 & 0.37 & 77.8 & $2,968 \pm 32$ & $3205.2 \pm 4.6$ & 7 & 0.25325 & 0.29 & 20.42 & 1.4 & 0.5848 & 1.3 & 0.977 \\
\hline 15.2 & 0.07 & 107 & 40 & 0.38 & 33.6 & $2,005 \pm 23$ & $2049 \pm 12$ & 2 & 0.12646 & 0.66 & 6,361 & 1.5 & 0.3648 & 1.3 & 0.897 \\
\hline 16.1 & 0.02 & 385 & 136 & 0.37 & 187.0 & $2,887 \pm 30$ & $3050.7 \pm 7.2$ & 5 & 0.2298 & 0.45 & 17.9 & 1.4 & 0.565 & 1.3 & 0.946 \\
\hline 17.1 & 0.06 & 68 & 101 & 1.53 & 21.6 & $2,031 \pm 24$ & $2051 \pm 13$ & 1 & 0.12656 & 0.72 & 6.46 & 1.6 & 0.3703 & 1.4 & 0.890 \\
\hline
\end{tabular}

Errors are 1-sigma. Error in standard calibration was $0.38 \%$ (not included in above errors but required when comparing data from different mounts).

${ }^{\mathrm{a}} \mathrm{Pbc}$ and $\mathrm{Pb}$ indicate the common and radiogenic portions, respectively.

b Common $\mathrm{Pb}$ corrected using measured ${ }^{204} \mathrm{~Pb}$.

mately in half. The mount surface was then polished to expose the grain interiors and photographed in order to obtain cathodo-luminescence (CL) images. Ion microprobe analyses were carried out using SHRIMP RG. Analytical methods and data treatment followed those described by Williams (1998) and Williams and Meyer
(1998). Common lead corrections were performed using the Cumming and Richards (1975) model. Uncertainties reported in Tables 1 and 2 for analyses are given at the $1 \sigma$ level, and final ages are quoted at $2 \sigma$ confidence level. The data have been processed using SQUID and Isoplot/Ex software (Ludwig, 2001a,b). 
ID-TIMS U-Pb analyses were carried out at the Geochronology Laboratory of the University of Brasília. Monazite grains were dissolved in concentrated $\mathrm{H}_{2} \mathrm{SO}_{4}$ $(5 \mu \mathrm{L})$, and titanite grains in concentrated $\mathrm{HF}$ and $\mathrm{HNO}_{3}$ (HF: $\mathrm{HNO}_{3}=4: 1$ ). A mixed ${ }^{205} \mathrm{~Pb}-{ }^{235} \mathrm{U}$ spike was used. Chemical extraction followed standard anion exchange technique, using Teflon micro columns, following procedures modified from Krogh (1973). $\mathrm{Pb}$ and $\mathrm{U}$ were loaded together on single Re filaments with $\mathrm{H}_{3} \mathrm{PO}_{4}$ and
Si gel for monazite analyses, and separately for titanite analyses. Isotopic analyses were carried out on a Finnigan MAT-262 multi-collector mass spectrometer equipped with secondary electron multiplier-ion counting. Procedure blanks for $\mathrm{Pb}$, at the time of analyses, were better than $15 \mathrm{pg}$. For data reduction and age calculation, PBDAT (Ludwig, 1993) and ISOPLOT-Ex (Ludwig, 2001a) were used. Errors for isotopic ratios are $2 \sigma$.

(a)

a)
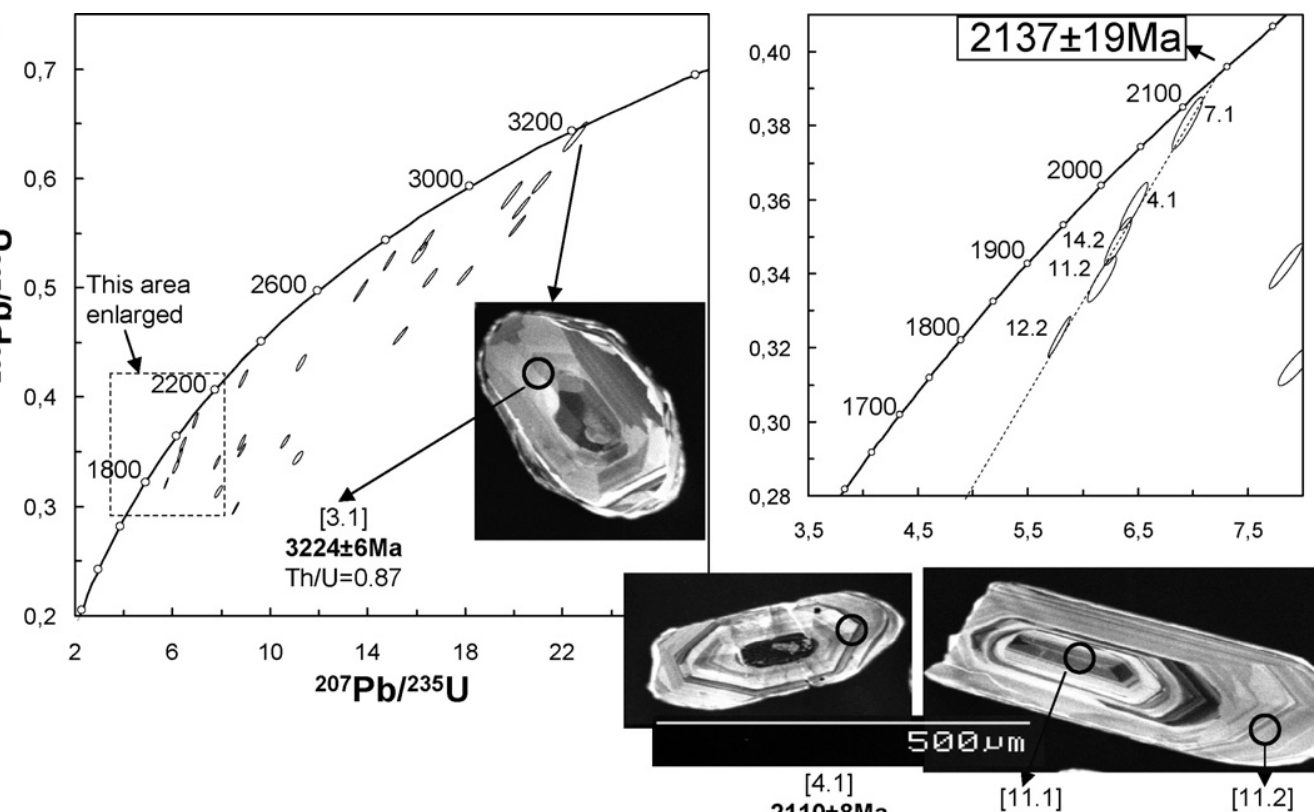

$2110 \pm 8 \mathrm{Ma}$

$\mathrm{Th} / \mathrm{U}=0.65$

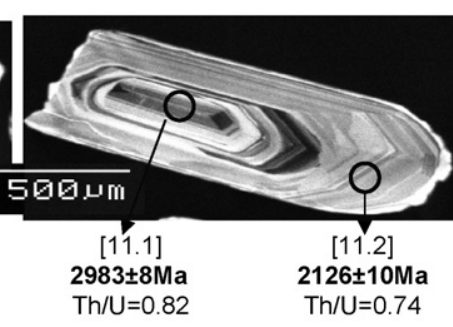

(b)

(10) os
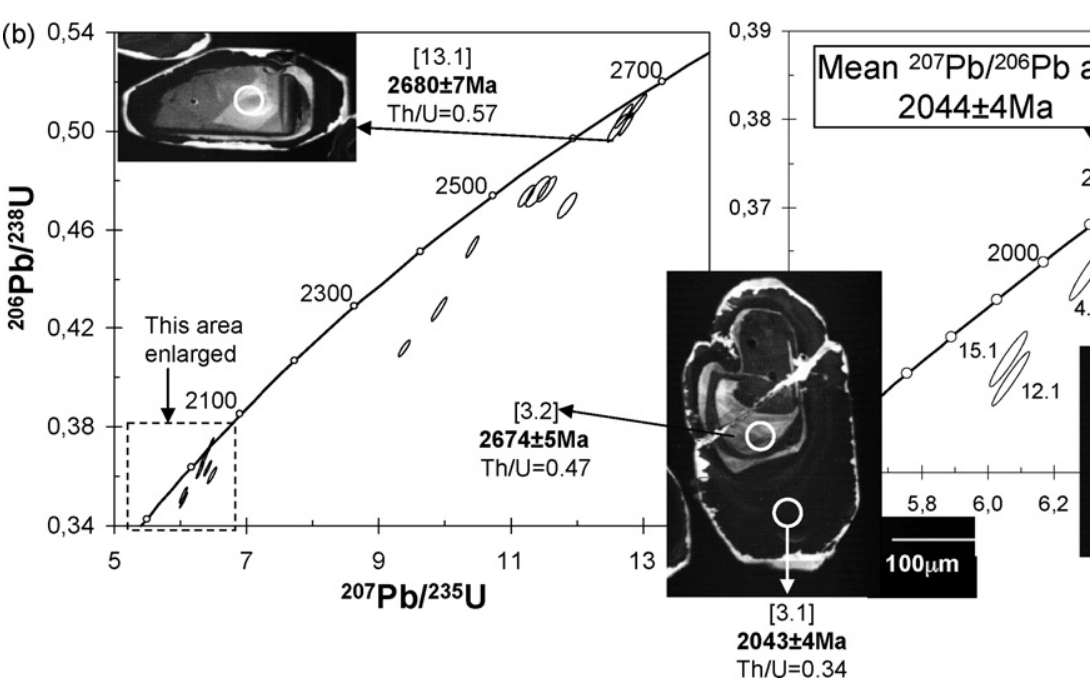

$2126 \pm 10 \mathrm{Ma}$
$\mathrm{Th} / \mathrm{U}=0.74$

Fig. 4. Concordia diagrams and CL images of zircon grains, Mantiqueira gneiss. Data-point error ellipses are 68.3\% confidence level. (4.a) Sample RP-1, five points discordia line, MSWD = 1.5; (4.b) sample LC-70; (4.c) sample LC-77; (4.d) sample LC-79, 10 points discordia line, MSWD=2.8; (4.e) sample LC-78, 17 points discordia line (14 zircon and 3 titanite points), MSWD = 2.1; (4.f) sample LC-67. 
(c)
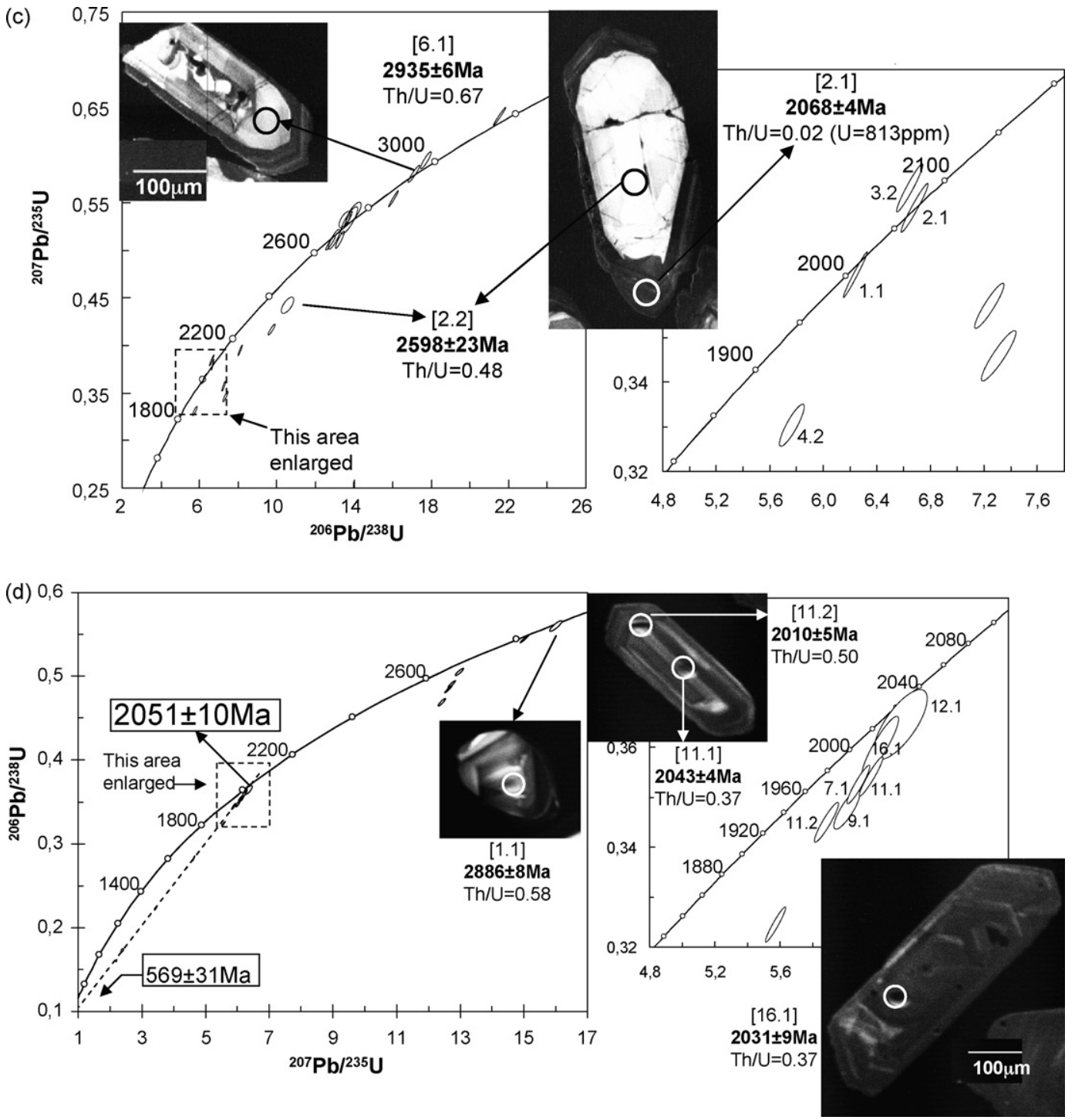

Fig. 4. (Continued).

\section{2. $U-P b$ data for the Mantiqueira Complex}

\subsubsection{Sample RP-01}

Twenty-six spot analyses were obtained on 16 zircon grains (Table 2), disclosing a large age spectrum with ${ }^{207} \mathrm{~Pb} /{ }^{206} \mathrm{~Pb}$ ages ranging from 2096 to $3263 \mathrm{Ma}$ (Fig. 4a). This is due to the preponderance of inherited zircon grains with varied morphological aspects, which yield discordant analysis essentially of Archean age. Many inherited grains display a magmatic overgrowth and an older core also magmatic-textured.

Crystallization age is given by four zircon grains displaying magmatic (oscillatory-zoned) overgrowths and inherited cores, and one homogeneous grain (without core/rim separation). Analyses of this grain (spot analysis 7.1) and the overgrowths (spot analyses 4.1, 11.2, $12.2,14.2)$ plot on a discordia line with an upperintercept age of $2137 \pm 19 \mathrm{Ma}$. The lower intercept is poorly constrained, but most zircon grains display a thin and bright luminescent outer rim in CL images that may be related to the Neoproterozoic metamorphic imprint.

The zircon ages obtained for this tonalite gneiss revealed a crystallization age of $2137 \pm 19 \mathrm{Ma}$ and the abundance of inherited zircon grains of Archean age. Analyses of inherited grains are in general highly discordant and yield a polymodal ${ }^{207} \mathrm{~Pb} /{ }^{206} \mathrm{~Pb}$ age distribution, what can be explained by a combination of different crystallization ages, and multiple episodes of lead loss plus 

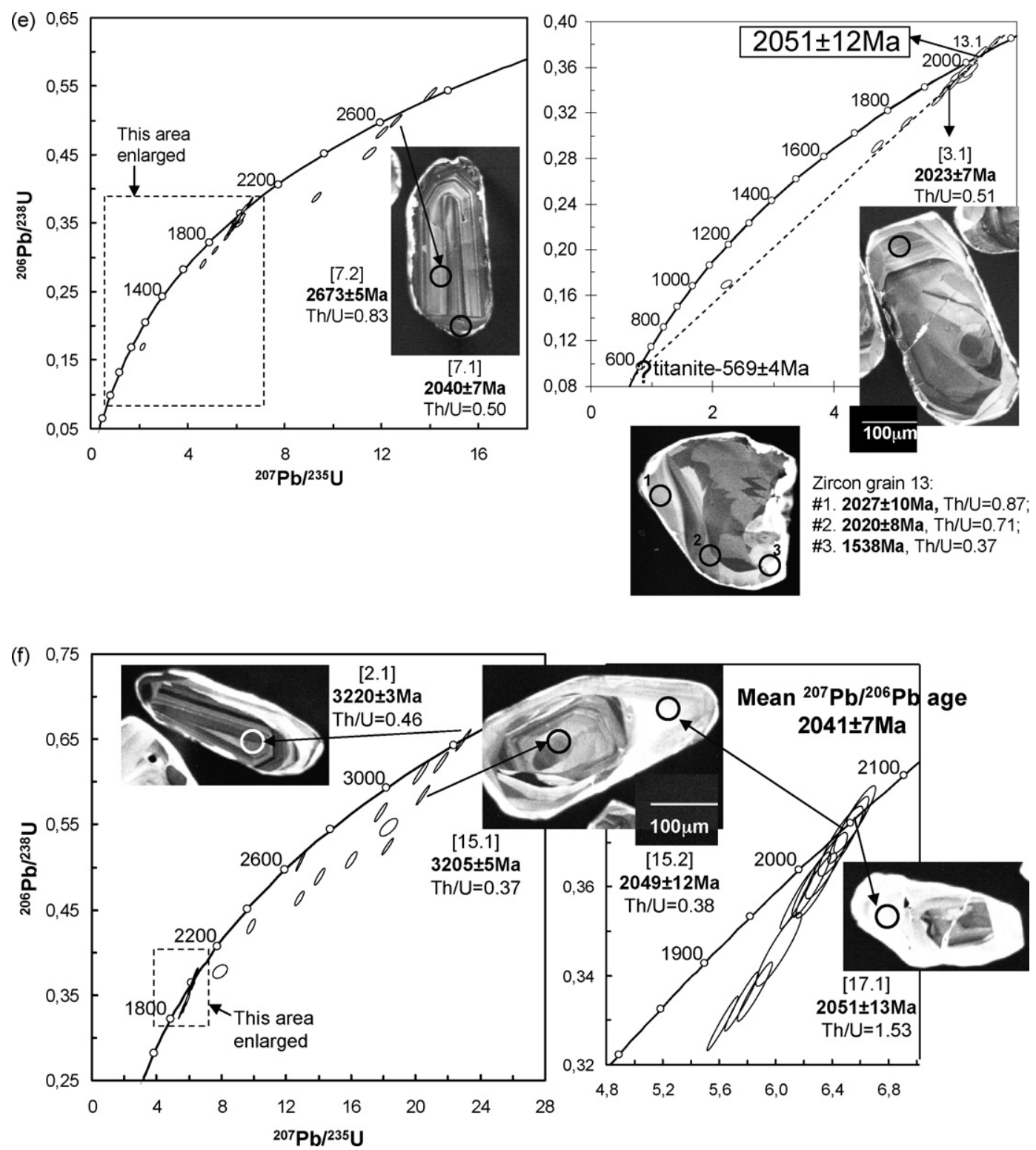

Fig. 4. (Continued).

recent lead loss. The same pattern is found for all samples of Mantiqueira gneiss discussed bellow.

\subsubsection{Sample $L C-70$}

Eighteen spot analyses were carried out on 15 zircon crystals (Table 2). Seven grains are subhedral and poorly faceted, displaying a core with oscillatory or complex-zoned magmatic texture, and a large low luminescent overgrow with a faint zoning. Thin rims with bright luminescence are observed around all crystals. The low luminescent overgrowths (spot analyses 1.2, 3.1, 4.1, 7.1, 11.1, 12.1, 15.1) have high $\mathrm{U}$ and high (magmatic) Th/U ratios with one single exception (11.1), which is the most U-enriched (1674 ppm) but has a low $\mathrm{Th} / \mathrm{U}$ ratio of 0.02 . The magmatic nature of overgrowths is also deduced by the fact that some inherited cores are irregular-shaped and display engulfment of overgrowth material, apparently due to a process of magmatic corrosion. The best estimate for this episode of zircon grow is given by the mean ${ }^{207} \mathrm{~Pb} /{ }^{206} \mathrm{~Pb}$ age for the more concordant analyses $(3.1,4.1,11.1)$ of $2044 \pm 4 \mathrm{Ma}$ (Fig. 4b). Slightly older spot analyses 1.2 and 7.1 probably represent mixed core/rim domains and were thus discarded. 
The magmatic age of this gneiss sample is assumed at $2044 \pm 4 \mathrm{Ma}$. Inherited cores and xenocrysts yield discordant Archean ages, except for a group of nearconcordant analyses $(1.1,3.2,10.1,13.1)$ with a mean ${ }^{207} \mathrm{~Pb} /{ }^{206} \mathrm{~Pb}$ age of $2685 \pm 12 \mathrm{Ma}$.

\subsubsection{Sample $L C-77$}

Twenty spot analyses were obtained on 14 zircon grains (Table 2) of morphologically distinct populations. Cathodoluminescence (CL) images show a dominant zircon type with length/width ratios of $3: 1$, bright luminescent cores and dark rims. Cores have high $\mathrm{Th} / \mathrm{U}$ ratios (0.4-0.7) and display magmatic oscillatory zoning. Seven spot analyses (spots 1.2, 3.1, 4.1, 7.1, 10.1, 13.1, 14.1) are almost concordant with a mean ${ }^{207} \mathrm{~Pb} /{ }^{206} \mathrm{~Pb}$ age of $2720 \pm 13 \mathrm{Ma}$, assumed as the magmatic event that crystallized the inherited cores. Of the four spot analyses of the dark rims (spots 1.1, 2.1,3.2, 4.2), three are concordant to near-concordant but spread along the concordia line with ${ }^{207} \mathrm{~Pb} /{ }^{206} \mathrm{~Pb}$ ages ranging from $2018 \pm 3$ and $2068 \pm 4$ Ma (Fig. 4c). These overgrowths owe their low luminescence in CL images to the very high U-content, up to $2426 \mathrm{ppm}$, and the low $\mathrm{Th} / \mathrm{U}$ ratios $(0.02)$ may be explained by this anomalous enrichment in $\mathrm{U}$ rather than by a metamorphic origin. In fact, the overgrowths must be melt-precipitated due to features like oscillatory zoning and the protruding of rim into the core. Therefore, a magmatic age between $2018 \pm 3$ and $2068 \pm 4 \mathrm{Ma}$ is assumed for gneiss sample LC-77.

Besides the ca. $2720 \mathrm{Ma}$ inherited zircon cores, older inherited ones are also present in this rock; one is concordant at $2935 \pm 6 \mathrm{Ma}$ (spot 6.1) and the other at $3145 \pm 3 \mathrm{Ma}$ (spot 5.2). A remaining group of highly discordant analyses refer to other Archean xenocrysts that underwent a combination of Paleoproterozoic and younger episodes of lead loss.

\subsubsection{Sample LC-79}

Eighteen spot analyses of 16 zircon crystals (Table 2) resulted in two age groups. One age group comprises inherited Archean zircon grains; two of them are almost concordant at 2828 and $2886 \mathrm{Ma}$. The largest group is represented by prismatic, euhedral zoned crystals (spot analyses $4.1,4.2,5.1,7.1,8.1,9.1,11.1,11.2,14.1$, 16.1) with low luminescence (high $U$ contents of 926 to $3935 \mathrm{ppm}$ ). Oscillatory zoning and $\mathrm{Th} / \mathrm{U}$ ratios ranging from 0.24 to 0.50 are indicative of a magmatic origin. A dark overgrowth of similar characteristics (spot 6.1), and a high luminescent subhedral zircon crystal with a complex-zoned magmatic texture (spot 12.1), also fall into this age group. Regression of these data yields a discordia line with an upper intercept age of $2051 \pm 10 \mathrm{Ma}$ and a less-precise lower intercept of $569 \pm 31 \mathrm{Ma}$ (Fig. 4d). The first is assumed as the magmatic age of the gneiss. The Neoproterozoic metamorphic event at ca. $569 \mathrm{Ma}$ led only to a lead-loss process, because the grains do not display any metamorphic rim.

\subsubsection{Sample $L C-78$}

Zircon grains can be divided into three populations: (1) prismatic medium-dark luminescent crystals with a well-developed oscillatory zoned magmatic texture; (2) zircon grains with a core similar to the previous type, but displaying a bright magmatic zoned overgrowth (Th/U ratios of $0.46-1.05)$; (3) anhedral grains displaying a complex-zoned texture and high $\mathrm{Th} / \mathrm{U}$ ratios (0.71-1.25). Five spot analyses of zircon grains of the first population and inherited cores of the second one (spot analyses 2.1, 7.2, 9.1, 10.1, 11.1) yield Archean ages.

Magmatic overgrowths (spot analyses 1.1, 3.1, 4.1, $5.1,6.1,7.1,10.2,12.1$ ), and zircon grains of the third group (spot analyses 8.1, 8.2, 13.1, 13.2, 13.3) define a linear array pointing to a Paleoproterozoic age (Table 2, Fig. 4e). Three U-Pb ID-TIMS analyses of titanite fractions are almost concordant with a mean age of $569 \pm 4 \mathrm{Ma}$ (Table 3 ). Regression of the titanite and zircon data gives an upper intercept age of $2051 \pm 12 \mathrm{Ma}$, and a lower intercept age of $570 \pm 36 \mathrm{Ma}$ (Fig. 4e). Zircon spot analysis 13.3 is not included in the age calculation because of very low $\mathrm{U}$ content and large errors on isotopic ratios.

The upper intercept age of $2051 \pm 12$ Ma can be interpreted as the magmatic age of the gneiss. Neoproterozoic metamorphic overprint, that is probably reflected by a thin bright luminescent outer rim displayed by most zircon crystals, is better constrained by the titanite age of $569 \pm 4 \mathrm{Ma}$.

\subsubsection{Sample LC-67}

The dominant zircon type is suhhedral to irregularshaped, displaying a zoned core and a large bright luminescent overgrowth. A thin outer rim of even higher luminescence can also be present. A second group comprises elongate to rounded grains displaying only the thin external rim. The zircon cores and this second group of zircon grains yield Archean ages. Most of the spot analyses are highly discordant and are scattered on the concordia diagram (Table 2, Fig. 4f). One inherited core is concordant at $3220 \pm 3 \mathrm{Ma}$ (spot analysis 2.1).

The rock crystallization age is given by nine spot analyses $(3.1,4.1,6.1,7.1,8.2,11.2,13.1,14.2$, 17.1) on the bright luminescent magmatic overgrowths, 


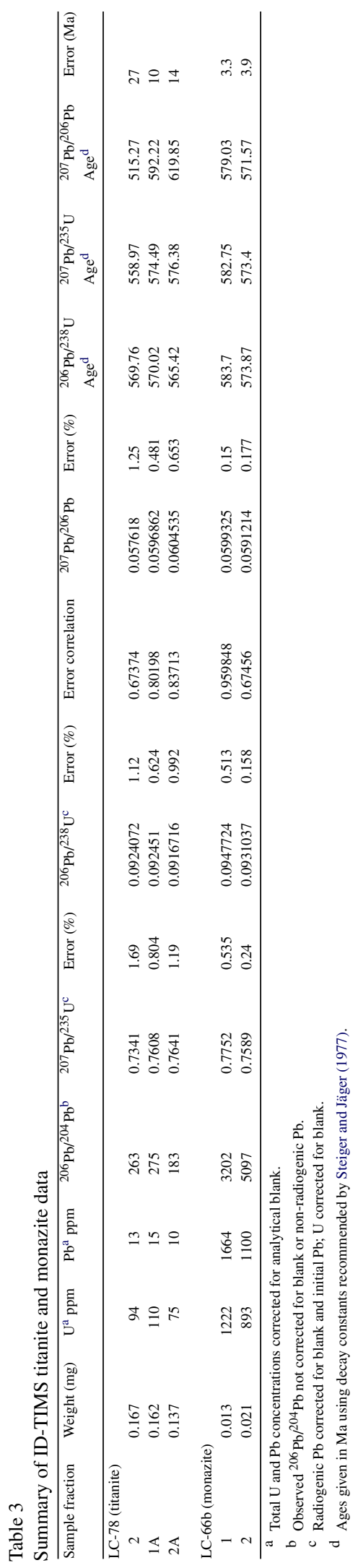

which display a faint oscillatory zoning and high $\mathrm{Th} / \mathrm{U}$ ratios (0.38-1.64). Analyses are collinear, concordant to $8 \%$ discordant and with a mean ${ }^{207} \mathrm{~Pb} /{ }^{206} \mathrm{~Pb}$ age of $2041 \pm 7$ Ma. Lead-loss must be related to the Neoproterozoic metamorphic episode.

\subsection{U-Pb data for the Juiz de Fora Complex}

\subsubsection{Sample LC-66a}

Zircon grains are prismatic to equal-sided, with rounded terminations. They are low luminescent showing a sector-zoned or oscillatory-zoned magmatic texture, and a thin bright luminescent rim. Although morphologically homogeneous, the zircon population has contrasting $\mathrm{Th} / \mathrm{U}$ ratios; one group has high (magmatic) ratios whilst the other has very low ratios due to a strong Th depletion. Metamorphic zircons of a granulite facies orthogneiss displaying a quite similar morphology and amount of Th depletion have been described elsewhere (Piuzana et al., 2003).

Eight spot analyses are discordant but colinear, defining a discordia line with an upper intercept age of $2113 \pm 20 \mathrm{Ma}$. Two analyses of grains with low $\mathrm{Th} / \mathrm{U}$ ratios (spot analyses $4.1,7.1$ ) plot above the discordia line. A regression of the six points representing zircon grains with typical magmatic high $\mathrm{Th} / \mathrm{U}$ ratios (spot analyses 1.1, 2.1, 3.1, 5.1, 6.1, 10.1) yields an upper intercept age of $2119 \pm 16 \mathrm{Ma}$, assumed as the best estimate for the gabbro-diorite magmatic age (Table 4, Fig. 5). The highgrade Paleoproterozoic metamorphic event is probably slightly younger than the intrusion age, and is responsible for the disturbance of the isotopic system of part of the zircon grains.

Two monazite grains from the late pink granite veins (sample LC-66b) were analyzed by ID-TIMS, and are concordant at ca. 584 and $574 \mathrm{Ma}$ (Table 3), thus providing a good constrain for the Neoproterozoic metamorphic overprint.

\subsubsection{Sample Ub-1}

Zircon population is composed of prismatic and equal-sided grains, presenting oscillatory zoned cores and a bright luminescent overgrowth. $\mathrm{Th} / \mathrm{U}$ ratios mostly above 0.3 for both cores and overgrowths are typically magmatic, but the latter show a strong U- and Thdepletion. Core/overgrowth boundary is quite irregular, with many features suggesting the engulfment of overgrowth material into the core. This is clearly seen in one of the zircon grains, together with the presence of a bright halo around a dark mineral inclusion within the core (Fig. 5b). Similar features have been already described in zircon crystals from migmatites, and the 
Table 4

Summary of SHRIMP U-Pb zircon data for Juiz de Fora gneiss samples

\begin{tabular}{|c|c|c|c|c|c|c|c|c|c|c|c|c|c|c|c|}
\hline Grain spot & $\%{ }^{206} \mathrm{Pbc}$ & $\mathrm{U}(\mathrm{ppm})$ & Th (ppm) & ${ }^{232} \mathrm{Th} /{ }^{238} \mathrm{U}$ & ${ }^{206} \mathrm{~Pb}^{\mathrm{a}}$ & ${ }^{206} \mathrm{~Pb} /{ }^{238} \mathrm{U}$ age ${ }^{\mathrm{b}}$ & ${ }^{207} \mathrm{~Pb} /{ }^{206} \mathrm{~Pb}$ age ${ }^{\mathrm{b}}$ & $\%$ discordant & ${ }^{207} \mathrm{~Pb}^{\mathrm{a}} / 206 \mathrm{~Pb}^{\mathrm{a}, \mathrm{b}}$ & $\pm \%$ & ${ }^{207} \mathrm{~Pb}^{\mathrm{a}} / 235 \mathrm{U}^{\mathrm{b}}$ & $\pm \%$ & ${ }^{206} \mathrm{~Pb}^{\mathrm{a}} / 238 \mathrm{U}^{\mathrm{b}}$ & $\pm \%$ & $\overline{\text { Error correction }}$ \\
\hline \multicolumn{16}{|c|}{ Sample LC-66a } \\
\hline 1.1 & 0.05 & 585 & 246 & 0.43 & 172.0 & $1,900 \pm 13$ & $2077.2 \pm 6.1$ & 9 & 0.1284764 & 0.35 & 6.0718254 & 0.8 & 0.3427637 & 0.8 & 0.912 \\
\hline 2.1 & 0.06 & 703 & 845 & 1.24 & 180.0 & $1,680 \pm 12$ & $2033.5 \pm 4.8$ & 17 & 0.1253356 & 0.27 & 5.1456066 & 0.8 & 0.2977563 & 0.8 & 0.944 \\
\hline 3.1 & 0.04 & 678 & 373 & 0.57 & 164.0 & $1,597 \pm 11$ & $2005.4 \pm 6.5$ & 20 & 0.1233643 & 0.37 & 4.7801732 & 0.8 & 0.2810302 & 0.7 & 0.896 \\
\hline 4.1 & 0.03 & 719 & 45 & 0.06 & 202.0 & $1,818 \pm 12$ & $1,982 \pm 9.1$ & 8 & 0.1217751 & 0.51 & 5.4706988 & 0.9 & 0.3258241 & 0.8 & 0.828 \\
\hline 5.1 & 0.03 & 407 & 598 & 1.52 & 118.0 & $1,874 \pm 12$ & $2,083 \pm 5.5$ & 10 & 0.1288995 & 0.32 & 5.9942908 & 0.8 & 0.3372758 & 0.8 & 0.923 \\
\hline 6.1 & 0.07 & 525 & 141 & 0.28 & 160.0 & $1,953 \pm 13$ & $2083.0 \pm 8.7$ & 6 & 0.1288995 & 0.49 & 6.2891893 & 0.9 & 0.3538686 & 0.7 & 0.834 \\
\hline 7.1 & 0.00 & 724 & 43 & 0.06 & 185.0 & $1,681 \pm 11$ & $1,946 \pm 9$ & 14 & 0.11933 & 0.52 & 4.9020078 & 0.9 & 0.297936 & 0.7 & 0.818 \\
\hline 8.1 & 0.02 & 857 & 66 & 0.08 & 245.0 & $1,854 \pm 12$ & $2067.9 \pm 4.0$ & 10 & 0.1277951 & 0.23 & 5.8708981 & 0.8 & 0.3331877 & 0.7 & 0.954 \\
\hline 9.1 & 0.00 & 1100 & 20 & 0.02 & 303.0 & $1,794 \pm 11$ & $2,044 \pm 3.8$ & 12 & 0.1260935 & 0.21 & 5.5771202 & 0.7 & 0.3207866 & 0.7 & 0.961 \\
\hline 10.1 & 1.36 & 487 & 631 & 1.34 & 136.0 & $1,789 \pm 75$ & $2,045 \pm 33$ & 13 & 0.1261447 & 1.87 & 5.5636943 & 5.2 & 0.3198845 & 4.8 & 0.932 \\
\hline \multicolumn{16}{|c|}{ Sample Ub-1 } \\
\hline 1.1 & 0.00 & 371 & 289 & 0.80 & 103.0 & $1,805 \pm 24$ & $2,011 \pm 12$ & 10 & 0.12378 & 0.69 & 5,514 & 1.7 & 0.3231 & 1.5 & 0.910 \\
\hline 2.1 & 0.52 & 23 & 14 & 0.61 & 2.6 & $792 \pm 28$ & $1,256 \pm 78$ & 37 & 0.0824 & 4.00 & 1,486 & 5.5 & 0.1307 & 3.8 & 0.688 \\
\hline 2.2 & 0.00 & 912 & 350 & 0.40 & 232.0 & $1,673 \pm 22$ & $1969.0 \pm 8.3$ & 15 & 0.12087 & 0.47 & 4,938 & 1.6 & 0.2963 & 1.5 & 0.955 \\
\hline 3.1 & 0.06 & 212 & 229 & 1.12 & 56.0 & $1,730 \pm 22$ & $2,033 \pm 10$ & 15 & 0.12529 & 0.59 & 5,319 & 1.6 & 0.3079 & 1.5 & 0.930 \\
\hline 3.2 & 0.03 & 363 & 102 & 0.29 & 113.0 & $1,994 \pm 25$ & $2089.7 \pm 6.3$ & 5 & 0.12939 & 0.36 & 6,469 & 1.5 & 0.3626 & 1.4 & 0.970 \\
\hline 4.1 & 0.04 & 411 & 603 & 1.52 & 97.9 & $1,575 \pm 20$ & $1,936 \pm 11$ & 19 & 0.11868 & 0.62 & 4,530 & 1.6 & 0.2768 & 1.5 & 0.920 \\
\hline 4.2 & 0.01 & 964 & 460 & 0.49 & 247.0 & $1,679 \pm 21$ & $2017.4 \pm 7.5$ & 17 & 0.1242 & 0.42 & 5,096 & 1.5 & 0.2976 & 1.4 & 0.958 \\
\hline 5.1 & 0.01 & 513 & 275 & 0.55 & 157.0 & $1,962 \pm 24$ & $2057.4 \pm 5.1$ & 5 & 0.12704 & 0.29 & 6,231 & 1.5 & 0.3557 & 1.4 & 0.980 \\
\hline 5.2 & 0.00 & 832 & 523 & 0.65 & 229.0 & $1,794 \pm 23$ & $2028.0 \pm 5.6$ & 12 & 0.12494 & 0.32 & 5,528 & 1.5 & 0.3209 & 1.5 & 0.978 \\
\hline 6.1 & 0.00 & 2524 & 1684 & 0.69 & 461.0 & $1,243 \pm 16$ & $1790.9 \pm 9.6$ & 31 & 0.10949 & 0.53 & 3,211 & 1.5 & 0.2127 & 1.4 & 0.939 \\
\hline 6.2 & 2.75 & 33 & 10 & 0.32 & 3.6 & $734 \pm 19$ & $1,120 \pm 80$ & 34 & 0.0769 & 9.20 & 1.28 & 9.6 & 0.1207 & 2.7 & 0.285 \\
\hline 7.1 & 0.05 & 159 & 67 & 0.44 & 28.2 & $1,205 \pm 17$ & $1,754 \pm 14$ & 31 & 0.10728 & 0.74 & 3,041 & 1.7 & 0.2056 & 1.5 & 0.898 \\
\hline 7.2 & 0.15 & 951 & 388 & 0.42 & 208.0 & $1,460 \pm 19$ & $1,907 \pm 11$ & 23 & 0.11673 & 0.61 & 4,093 & 1.5 & 0.2543 & 1.4 & 0.920 \\
\hline 8.1 & 0.14 & 131 & 117 & 0.92 & 41.2 & $2,004 \pm 28$ & $2,064 \pm 14$ & 3 & 0.1275 & 0.80 & 6.41 & 1.8 & 0.3647 & 1.6 & 0.898 \\
\hline 8.2 & 0.20 & 652 & 58 & 0.09 & 87.5 & $934 \pm 17$ & $1,636 \pm 19$ & 43 & 0.1007 & 1.00 & 2,163 & 2.2 & 0.1559 & 2.0 & 0.895 \\
\hline 9.1 & 0.04 & 255 & 80 & 0.32 & 73.4 & $1,863 \pm 24$ & $2045.9 \pm 7.6$ & 9 & 0.12621 & 0.43 & 5,832 & 1.5 & 0.3351 & 1.5 & 0.960 \\
\hline 10.1 & 0.13 & 264 & 177 & 0.69 & 79.8 & $1,939 \pm 25$ & $2046.1 \pm 8.4$ & 5 & 0.12623 & 0.48 & 6,107 & 1.6 & 0.3509 & 1.5 & 0.952 \\
\hline 11.1 & 0.01 & 1030 & 897 & 0.90 & 341.0 & $2,104 \pm 25$ & $2078.9 \pm 4.0$ & -1 & 0.1286 & 0.22 & 6,845 & 1.4 & 0.386 & 1.4 & 0.988 \\
\hline 12.1 & 0.15 & 70 & 43 & 0.63 & 20.4 & $1,869 \pm 28$ & $2,053 \pm 30$ & 9 & 0.1267 & 1.70 & 5.88 & 2.4 & 0.3363 & 1.7 & 0.708 \\
\hline 13.1 & 0.00 & 1708 & 1338 & 0.81 & 517.0 & $1,947 \pm 24$ & $2046.0 \pm 3.3$ & 5 & 0.12622 & 0.19 & 6,136 & 1.4 & 0.3526 & 1.4 & 0.991 \\
\hline
\end{tabular}

Errors are 1-sigma. Error in standard calibration was $0.31 \%$ (not included in above errors but required when comparing data from different mounts).

${ }^{\text {a }} \mathrm{Pbc}$ and $\mathrm{Pb}$ indicate the common and radiogenic portions, respectively.

${ }^{b}$ Common $\mathrm{Pb}$ corrected using measured ${ }^{204} \mathrm{~Pb}$. 
(a)
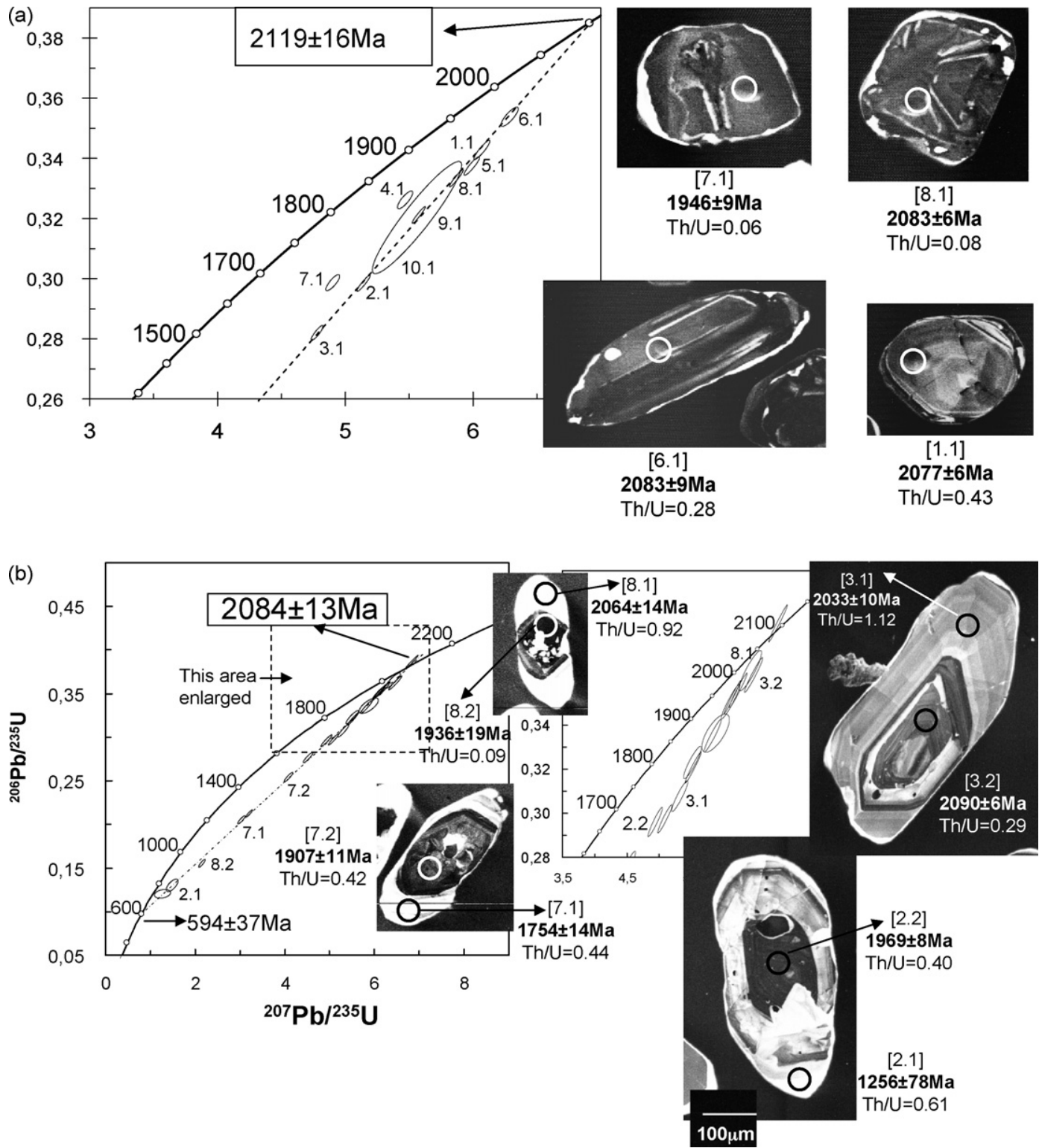

Fig. 5. Concordia diagrams and CL images of zircon grains, Juiz de Fora gneiss. Data-point error ellipses are 68.3\% confidence level. (5.a) Sample LC-66a, eight points discordia line, MSWD =1.7; (5.b) sample Ub-1 nineteen points discordia line, $\mathrm{MSWD}=3.1$.

overgrowth forming mechanism attributable to anatectic melting-precipitation of new zircon material (e.g., Silva et al., 2005b).

Twenty spot analyses of core and overgrowth were obtained on 13 zircon grains (Table 4), and the data define a discordia line with an upper intercept age of $2084 \pm 13 \mathrm{Ma}$ and a lower intercept age of $594 \pm 37 \mathrm{Ma}$ (Fig. 5b). When data for cores and overgrowths are regressed separately the same upper intercept age within errors is obtained. Spot analyses are concordant to highly discordant and the overgrowths in general experienced a more severe lead loss during the Neoproterozoic metamorphic overprint.

The coincidence of core and overgrowth ages at $2084 \pm 13 \mathrm{Ma}$ may be explained by migmatization shortly following the pluton intrusion probably during a syn-collisional stage.

\section{Discussion}

A summary of $\mathrm{U}-\mathrm{Pb}$ data together with $\mathrm{Nd}$ signatures are presented in Table 5. Samples of Mantiqueira gran- 
Table 5

Summary of isotopic data

\begin{tabular}{|c|c|c|c|c|c|c|}
\hline Sample & Gneiss composition & Sample location & Magmatic age & Metamorphic age(s) & $T_{\mathrm{DM}}(\mathrm{Ga})$ & $\varepsilon_{\mathrm{Nd}(\mathrm{t})}$ \\
\hline \multicolumn{7}{|c|}{ Mantiqueira Complex } \\
\hline RP-1 & Tonalite & $\begin{array}{l}\text { Quarry in the vicinity of Alto } \\
\text { Rio Doce }\end{array}$ & $2137 \pm 19$ & & 3.0 & -13.0 \\
\hline LC-70 & Granodirite & $\begin{array}{l}\text { Road cut between Mariana } \\
\text { and Ponte Nova }\end{array}$ & $2044 \pm 4$ & & 2.9 & -9.0 \\
\hline LC-77 & Granodiorite & $\begin{array}{l}\text { Road cut close to São } \\
\text { Domingos do Prata. }\end{array}$ & $2018 \pm 3$ to $2068 \pm 4$ & & 3.1 & -11.4 \\
\hline LC-79 & Granite & $\begin{array}{l}\text { Quarry in the town of } \\
\text { Coronel Fabriciano }\end{array}$ & $2051 \pm 10$ & $569 \pm 31$ & 2.9 & -9.6 \\
\hline LC-78 & Granodiorite & $\begin{array}{l}\text { Outcrop at the margin of } \\
\text { Doce river, within the Natural } \\
\text { Rio Doce Park }\end{array}$ & $2051 \pm 12$ & $570 \pm 36(569 \pm 4)$ & 3.2 & -12.0 \\
\hline LC-67 & Granodiorite & Road cut near Rio Casca & $2041 \pm 7$ & & 3.2 & -12.0 \\
\hline \multicolumn{7}{|c|}{ Juiz de Fora Complex } \\
\hline LC-66 & Norite & Abre Campo quarry & $2119 \pm 16$ & $584-574$ & 2.3 & -0.4 \\
\hline Ub-1 & Enderbite & Astolfo Dutra quarry & $2084 \pm 13$ & $594 \pm 37$ & 2.6 & -3.5 \\
\hline
\end{tabular}

odiorite and granite gneiss crystallized at ca. $2050 \mathrm{Ma}$, and are characterized by the abundance of inherited Archean zircon grains, $T_{\mathrm{DM}}$ model ages ranging from 2.9 to $3.2 \mathrm{Ga}$, and strongly negative $\varepsilon_{\mathrm{Nd}(\mathrm{t})}(-9$ to -12$)$. These data indicate that the Archean crust was the main source of magmas that gave origin to the gneiss protholits. The preservation of large volumes of inherited zircon crystals in crustal anatectic melts has been explained by low melting temperatures (Watson and Harrison, 1983), as is the case for diatexite migmatites (Tikhomirova, 2002). Sample RP-1 crystallized at ca. $2137 \mathrm{Ma}$, is tonalitic in composition and also carries a large amount of inherited Archean zircon grains. It yields a strongly negative $\varepsilon_{\mathrm{Nd}(\mathrm{t})}$ of -13 , and should derive from a crust-contaminated magma at an active-margin setting.

These results are in agreement with previous $\mathrm{U}-\mathrm{Pb}$ SHRIMP data for three Mantiqueira gneiss samples, which yielded crystallization ages at $2102-2058 \mathrm{Ma}$, with inherited zircon nuclei dated at $2180-2160 \mathrm{Ma}$ (Silva et al., 2002). It was proposed that inherited zircon nuclei derived from partial melting of arcrelated granitoids during syn-collisional magmatism at 2102-2058 Ma. A banded gneiss dated by Heilbron et al. (2001) records a metamorphic event at $2040 \pm 47 \mathrm{Ma}$, and its crystallization age at $2203 \pm 17 \mathrm{Ma}$ suggests a complex and protracted evolution for the magmatic $\operatorname{arc}(\mathrm{s})$ plutons included into the Mantiqueira Complex.

The most striking feature of the results for Mantiqueira gneisses is the spread out of the analytical data observed in all of the concordia diagrams, which simulates the distribution pattern of zircon grains from metasedimentary rocks. In fact, this pattern is essentially a consequence of the polycyclic history of the
Mantiqueira Complex. Most analyses of inherited zircon grains are moderately to highly discordant due to multiple episodes of lead loss during the Paleo and Neoproterozoic, resulting in a large spread of ${ }^{207} \mathrm{~Pb} /{ }^{206} \mathrm{~Pb}$ ages. Besides, for some of the samples inherited zircon grains and cores may fall into more than one age group, a feature that can be found in other continental arc-related plutonic rocks (for example. some tonalite gneiss and granodiorite of the Minto block, northeastern Superior Province; Percival et al., 2001).

Juiz de Fora gneiss samples have no inherited zircons. The gabbro-dioritic rock (sample LC-66a) yields a juvenile $\mathrm{Nd}$ signature, with a $T_{\mathrm{DM}}$ model age of $2.3 \mathrm{Ga}$ and a $\varepsilon_{\mathrm{Nd}(\mathrm{t})}$ value close to zero, whilst the enderbitic gneiss (sample Ub-1) has a Late Archean $T_{\mathrm{DM}}$ model age $(2.6 \mathrm{Ga})$ and a negative $\varepsilon_{\mathrm{Nd}(\mathrm{t})}$ value of -3.5 . For the latter, a mixed source of juvenile Paleoproterozoic material with contribution of Archean crust material is suggested. Intrusion ages for Juiz de Fora gneiss, from this and previous studies fall into the 2134-2084 Ma time range, with the exception of one older age of $2195 \pm 15 \mathrm{Ma}$ (Silva et al., 2002).

Isotopic evidence, specially for Juiz de Fora gneiss samples, suggest the studied units underwent a migmatization process during the Paleoproterozoic. This is in agreement with field observation that the gneissic banding results from the transposition of an earlier banded structure during the Brasiliano Orogeny (Fig. 3b).

\subsection{Correlation with the West Congo Belt}

The basement of the West Congo Belt is represented by the Paleoproterozoic Kimezian Supergroup, to the 
west and north, and Archean complexes, to the southeast (Fig. 1). The Kimezian Supergroup is essentially a series of metasedimentary rocks and consists of paragneiss, amphibolite, migmatite, and minor quartzite and marble, metamorphosed around $2 \mathrm{Ga}$ (Delhal and Ledent, 1976; Vicat and Pouclet, 2000; Tack et al., 2001). Deformed granitoids dated at ca. $2.0 \mathrm{Ga}$ have been interpreted as plutons of a Paleoproterozoic continental magmatic arc (Maurin et al., 1990, 1991; Vicat and Pouclet, 2000). The Paleoproterozoic basement was reworked during the Pan African orogeny under greenschist facies conditions to the east (i.e., towards the foreland of the West Congo Belt), and under amphibolite facies conditions to the west (i.e., to the westernmost part of the West Congo Belt).

$\mathrm{U}-\mathrm{Pb}$ data for Kimezian rocks comes from ID-TIMS analyses on unabraded zircon fractions, and analytical points are usually very discordant resulting in not well-constrained upper-intercept ages for crystallization events. Two migmatitic gneisses (Mpozo-Tombagadio and Boma migmatites) yielded an age of 2150-2125 Ma, interpreted as the migmatization age (Delhal and Ledent, 1976). Dated plutonic bodies are

- Guéna gneiss, a banded biotite gneiss tonalitic in composition, dated at 2014 $\pm 56 \mathrm{Ma}$ (Maurin et al., 1991); it has an Archean $T_{\mathrm{DM}}$ model age $(2.6 \mathrm{Ga})$ and negative $\varepsilon_{\mathrm{Nd}(\mathrm{t})}$.values ranging from -4.8 to -6.2 (Djama et al., 1992).

- Les Saras granodiorite, dated at $2000 \pm 80 \mathrm{Ma}$ (Maurin et al., 1990).

- Fougamou granitoid, comprising porphiritic rocks ranging in composition from granite to diorite, and dated at 1915+10/-9 Ma (Caen-Vachette et al., 1988).

- The Gombou massif, composed of granodiorite gneiss, and with a less-deformed facies dated at $2083+27 /-25 \mathrm{Ma}$ (Caen-Vachette et al., 1988).

- Vista Alegre granodiorite, dated at $1940 \mathrm{Ma}$ (Cahen et al., 1979).

- Mpozo syenite, dated at $1960 \mathrm{Ma}$ (Delhal and Ledent, 1978).

Les Saras and Guéna gneissic plutons are interpreted as calc-alkaline intrusions related to a magmatic arc built up at a continental margin in response to subduction of oceanic lithosphere under an Archean craton to the east (Maurin et al., 1990, 1991). Vicat and Pouclet (2000) also suggests a continental, active-margin setting for the calcalkaline to alkaline, Paleoproterozoic (2083-1915 Ma) magmatism recorded in the basement of the West Congo Belt, which was produced by partial melting of Archeanaged protholits.
A correlation arises from the available data: granitoid plutons of the Mantiqueira Complex and the West Congo Belt basement are probably related to two orogens that evolved along the margins of the Archean nuclei of São Francisco and Congo cratons. Orogenic evolution was diachronic; magmatism in the Mantiqueira Complex ended at ca. $2041 \mathrm{Ma}$, but extended until ca. $1915 \mathrm{Ma}$ in the Kimezian Supergroup of the West Congo Belt basement. The Juiz de Fora Complex seems to have no correlative in the basement of the West Congo Belt.

\section{Final remarks and evolutionary model}

All directions refers to the present-day position of South America and Africa.

A Paleoproterozoic belt (the West Central African Belt, Feybesse et al., 1998) extends along the western side of the Congo Craton, and is interpreted as a result of the collision between the São Francisco-Nigerian shield and a former Congo megacraton (Lerouge et al., 2006). This Paleoproterozoic belt represents the external sector of a larger orogen whose internal zones are preserved in the Itabuna-Salvador-Curaçá belt that runs along the eastern side of the São Francisco Craton facing the Atlantic Ocean (Ledru et al., 1994; Barbosa and Sabaté, 2004; Lerouge et al., 2006). Evolution of the Brasiliano-Pan African Araçuaí-West Congo Orogen disrupted and deeply reworked a segment of this Paleoproterozoic orogen to the south of the Itabuna-Salvador-Curaçá belt and its African counterpart. Its fragments are now the basement of the Araçuaí-West Congo Orogen, consisting of the following rock assemblages associated to distinct tectonic settings:

(1) The Mantiqueira Complex mainly comprises the plutonic rocks generated in an orogen that evolved along the Archean margin of the São Francisco Paleocontinent, approximately between 2180 and $2041 \mathrm{Ma}$.

(2) The Juiz de Fora Complex probably evolved within an oceanic magmatic arc setting, or on a very stretched continental crust, between 2195 and 2084 Ma.

(3) The Kimezian Supergroup, a series of metasedimentary rocks of unknown depositional age, metamorphosed during the Eburnean Orogeny.

(4) Calc-alkaline to alkaline granitoids evolved in a similar setting as the Mantiqueira Complex, but along the Archean margin of the Congo Paleocontinent, from 2083 to $1915 \mathrm{Ma}$. 
ca. $2180-2100 \mathrm{Ma}$
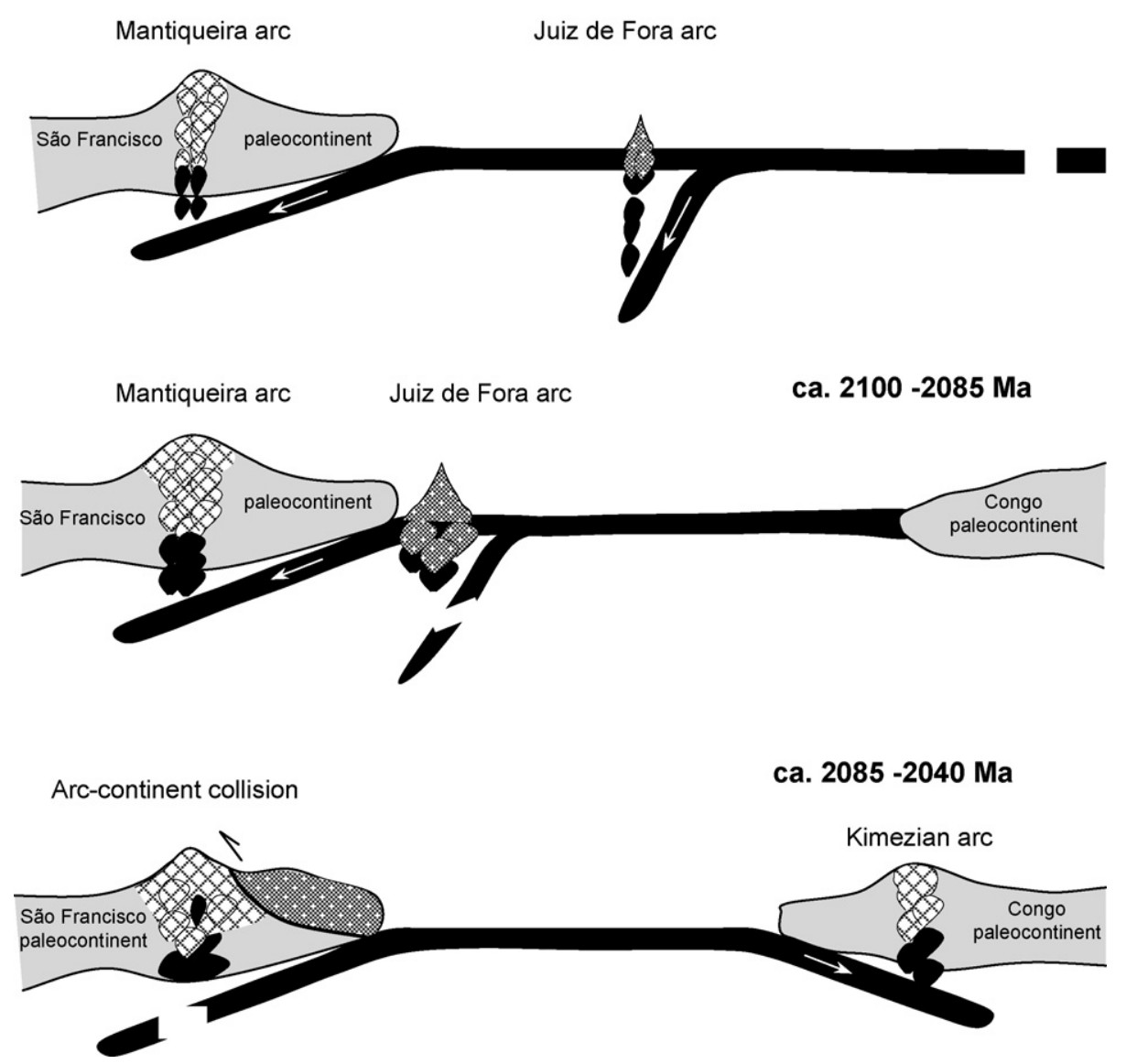

Fig. 6. Cartoon illustrating the possible scenarios for the evolution of Paleoproterozoic magmatic arcs in the São Francisco and Congo paleocontinents.

The presented data and interpretations of tectonic environments encourage the authors to offer a hypothesis in order to inspire future works on the evolution of the focused Paleoproterozoic basement terranes, as follows (Fig. 6):

- If the Juiz de Fora Complex is a remnant of an island arc, the model requires an intra-oceanic subduction zone and also implies that, much probably, a very large ocean separated the São Francisco and Congo paleocontinents during this stage (ca. 2180-2100 Ma). The Mantiqueira magmatic arc would be in part coeval to the Juiz de Fora arc, but developed on the eastern active margin of the São Francisco Paleocontinent.

- Evidence from zircon data suggest a collision between the Juiz de Fora arc and the São Francisco Paleocontinent in the interval of ca. 2100-2085 Ma.

- Orogenic, calc-alkaline magmatism would have begun in the Kimezian basement at ca. 2083 Ma. This requires subduction to the east, under the active mar- gin of the Congo Paleocontinent. The stage from $2085 \mathrm{Ma}$ to $2040 \mathrm{Ma}$ would record the last calcalkaline magmatism in the Mantiqueira arc in a syn- to late collisional tectonic setting. The younger (ca. 1915 Ma) alkaline plutons of the Kimezian basement could represent the post-collisional magmatism that followed the amalgamation of the São Francisco-Congo Paleocontinent.

\section{Acknowledgments}

C.M. Noce is grateful to the Fundação de Desenvolvimento da Pesquisa de Minas Gerais (FAPEMIG) for funding the analytical work at Research School of Earth Sciences (ANU). C.M. Noce and A.C. PedrosaSoares thank $\mathrm{CNPq}$ for continuous support. Simone Gioia and Márcio Pimentel (Universidade de Brasília) are thanked for Sm-Nd data, and Luc Tack (Africamuseum, Belgium) for his help with some references. Fernando Alkmim is gratefully acknowledged for criti- 
cal discussion. Two anonymous reviewers of Prec Res. are thanked for their useful comments and criticism.

\section{References}

Alkmim, F.F., Marshak, S., 1998. The Transamazonian orogeny in the Quadrilátero Ferrífero, Minas Gerais Brazil: Paleoproterozoic Collision and Collapse in the Souhtern São Francisco Craton region. Precamb. Res. 90, 29-58.

Alkmim, F.F., Marshak, S., Pedrosa-Soares, A.C., Peres, G.G., Cruz, S.C.P., Whittington, A., 2006. Kinematic evolution of the Araçuaí-West Congo orogen in Brazil and Africa: Nutcracker tectonics during the Neoproterozoic assembly of West Gondwana. Precamb. Res. 149, 43-64.

Barbosa, J.S.F., Sabaté, P., 2004. Archean and Paleoproterozoic crust of the São Francisco Craton, Bahia Brazil: geodynamic features. Precamb. Res. 133, 1-27.

Brito Neves, B.B., Campos Neto, M.C., Fuck, R., 1999. From Rodinia to Western Gondwana: an approach to the Brasiliano-Pan African cycle and orogenic collage. Episodes 22, 155-199.

Caen-Vachette, M., Vialette, Y., Bassot, J.-P., Vidal, P., 1988. Apport de la géochronologie isotopique à la connaissance de la géologie gabonaise. Chronique Recherche Minière 491, 35-54.

Cahen, L., Kröner, A., Ledent, D., 1979. The age of the Vista Alegre pluton and its bearing on the reinterpretation of the Precambrian geology of Northern Angola. Ann. Soc. Géol. Belgique 102, 265-275.

Costa, A.G., 1998. The granulite-facies rocks of the northern segment of the Ribeira Belt, eastern Minas Gerais, SE Brazil. Gondwana Res. 1, 367-372.

Cumming, G.L., Richards, J.R., 1975. Ore lead isotope ratios in a continuously changing Earth. Earth Planet. Sci. Lett. 28, 155-171.

D'Agrella Filho, M.S., Pacca, I.I.G., Trindade, R.I.F., Teixeira, W., Raposo, M.I.B., Onstott, T.C., 2004. Paleomagnetism and ${ }^{40} \mathrm{Ar}^{39} \mathrm{Ar}$ ages of mafic dykes from Salvador (Brazil): new constraints on the São Francisco Craton APW path between 1080 and 1010 Ma. Precamb. Res. 132, 55-77.

Delgado, I.M., Souza, J.D., Silva, L.C., Silveira Filho, N.C., Santos, R.A., Pedreira, A.J., Guimarães, J.T., Angelim, L.A.A., Vasconcelos, A.M., Gomes, I.P., Lacerda Filho, J. V, Valente, C.R., Perrotta, M.M., Heineck, C.A., 2003. Escudo Atlântico. In: Bizzi, L.A., Schobbenhaus, C., Vidotti, M., Gonçalves, J.H. (Eds.), Geologia, Tectônica e Recursos Minerais do Brasil. Editora Universidade de Brasília/CPRM, Brasília, pp. 227-334 (1 DVD).

De la Roche, H., Leterrier, J., Grande Claude, P., Marchal, M., 1980. A classification of volcanic and plutonic rocks using R1-R2 diagrams and major element analyses-its relationship and current nomenclature. Chem. Geol. 29, 183-210.

Djama, L.M., Leterrier, J., Michard, A., 1992. Pb, Sr and Nd isotope study of the basement of the Mayumbian Belt (Guéna gneisses and Mfoubu granite, Congo): implications for crustal evolution in Central Africa. J. African Earth Sci. 14, 227-237.

Delhal, J., Ledent, D., 1976. Age et évolution comparée des gneiss migmatitiques pré-zadiniens des régions de Boma et de MpozoTombagadio (Bas-Zaire). Ann. Soc. Géol. Belgique 99, 165-187.

Delhal, J., Ledent, D., 1978. Données géochronologiques sur lê socle précambrien de l'ouest du Congo. Ann. Soc. Géol. Belgique 99, 165-187.

DeWit, M., Jeffery, M., Bergh, H., Nicolaysen, L., 1988. Geologic Map of Sectors of Gondwana. AAPG and University of Witwatersrand, Tulsa.
Duarte, B.P., Figueiredo, M.C.H., Campos Neto, M., Heilbron, M., 1997. Geochemistry of the Granulite Fácies Orthogneisses of Juiz de Fora Complex, Central Segment of Ribeira Belt, Southeastern Brazil. Rev. Bras. Geociênc. 27, 67-82.

Duarte, B.P., Valente, S.C., Heilbron, M., Campos Neto, M.C., 2004. Petrogenesis of the orthogneisses of the Mantiqueira Complex, Central Ribeira Belt, SE Brazil: an Archaean to Paleoproterozoic basement unit reworked during the Pan-African Orogeny. Gondwana Res. 7, 437-450.

Duarte, B.P., Heilbron, M., Ragatky, D., Valente, S.C., 2005. Mantiqueira and Juiz de Fora Complexes: reworked basement units within a Western Gondwana mobile belt in Brazil. In: Abstracts, Gondwana 12. Academia Nacional de Ciências, Mendoza, Argentina, p. 142.

Feybesse, J.L., Johan, V., Triboulet, C., Guerrot, C., Mayaga-Mikolo, F., Bouchot, V., Eko N'dong, J., 1998. The West Central African belt: a model of 2.5-2.0 Ga accretion and two-phase orogenic evolution. Precamb. Res. 87, 161-216.

Figueiredo, M.C.H., Teixeira, W., 1996. The Mantiqueira Metamorphic Complex, eastern Minas Gerais State: preliminary geochronological and geochemical results. Anais Acad. Bras. Ciênc. 68, 223-246.

Fischel, D.P., Pimentel, M.M., Fuck, R.A., Costa, A.G., Rosière, C.A., 1998. Geology and Sm-Nd isotopic data for the Mantiqueira and Juiz de Fora Complexes (Ribeira Belt) in the Abre Campo-Manhuaçu region, Minas Gerais, Brazil. In: Abstracts, 14th International Conference on Basement Tectonics, Ouro Preto, Brazil, pp. 21-23.

Frimmel, H.E., Tack, L., Basei, M.S., Nutman, A.P., Boven, A., 2006. Provenance and chemostratigraphy of the Neoproterozoic West Congolian Group in the Democratic Republic of Congo. J. Afr. Earth Sci. 46, 221-239.

Heilbron, M., Machado, N., Duarte, B.P., 2001. Evolution of the Paleoproterozoic Transamazonian Orogen in SE Brazil: a view from the Neoproterozoic Ribeira Belt. In: Abstracts, GAC-MAC Joint Annual Meeting, St. Johns, Canada, vol. 26, p. 61.

Krogh, T., 1973. A low contamination method for hydrothermal decomposition of zircon and extraction of $\mathrm{U}$ and $\mathrm{Pb}$ for isotopic age determination. Geochim. Cosmochim. Acta 37, 488-494.

Ledru, P., Johan, V., Milési, J.P., Tegyey, M., 1994. Markers of the last stages of the Palaeoproterozoic collision: evidence for a 2 Ga continent involving circum-South Atlantic provinces. Precamb. Res. 69, 169-191.

Lerouge, C., Cocherie, A., Toteu, S.F., Penaye, J., Milési, J.-P., Tchameni, R., Nsifa, E.N., Fanning, M., Deloule, E., 2006. Shrimp $\mathrm{U}-\mathrm{Pb}$ zircon age evidence for Paleoproterozoic sedimentation and 2.05 syntectonic plutonism in the Nyong Group, South-Western Cameroon: consequences for the Eburnean-Transamazonian belt of NE Brazil and Central Africa. J. African Earth Sci. 44, 413-427.

Ludwig, K.R., 1993. PBDAT. A computer program for processing $\mathrm{Pb}-\mathrm{U}-\mathrm{Th}$ isotope data. USGS Open File Report 88-542, $34 \mathrm{pp}$.

Ludwig, K.R., 2001a. User's Manual for Isoplot/EX Version 2.47. A Geochronological Toolkit for Microsoft Excel. Berkeley Geochronological Center, Special Publication 1a, 55 pp.

Ludwig, K.R., 2001b. SQUID 1.02. A User's Manual. Berkeley Geochronological Center, Special Publication 2, 19 pp.

Machado, N., Valladares, C., Heilbron, M., Valeriano, C., 1996. U-Pb geochronology of the central Ribeira belt (Brazil) and implications for the evolution of the Brazilian Orogeny. Precamb. Res. 69, 347-361.

Maurin, J.-C., Mpemba-Bony, J., Pin, C., Vicat J.-P., 1990. La granodiorite de Les Saras un témoin de magmatisme éburnéen $(2 \mathrm{Ga})$ 
au sein de la chaine panafricaine du Mayombe (Congo). Compte Rendu Acad. Sci., Paris 310, 571-575, 96, 49-54.

Maurin, J.-C., Boudzoumou, F., Djama, L.M., Gioan, P., Michard, A., Mpemba-Boni, J., Peucat, J.-J., Pin, C., Vicat, J.-P., 1991. La chaine protérozoique ouest-congolienne et son avant-pays au Congon: nouvelles données géochronologiques et structurales, implications en Afrique centrale. Compte Rendu Acad. Sci., Paris $312,1327-1334$.

Noce, C.M., Teixeira, W., Quéméneur, J.J.G., Martins, V.T.S., Bolzachini, E., 2000. Isotopic signatures of Paleoproterozoic granitoids from southern São Francisco Craton and implications for the evolution of the Transamazonian Orogeny. J. South Am. Earth Sci. 13, 225-239.

Pedrosa-Soares, A.C., Noce, C.M., Wiedemann, C.M., Pinto, C.P., 2001. The Araçuaí-West Congo orogen in Brazil: An overview of a confined orogen formed during Gondwanland assembly. Precamb. Res. 110, 307-323.

Percival, J.A., Stern, R.A., Skulski, T., 2001. Crustal growth through successive arc magmatism: reconnaissance U-Pb SHRIMP data from the northeastern Superior Province, Canadá. Precamb. Res. 109, 203-238.

Peres, G.G., Alkmim, F.F., Jordt-Evangelista, H., 2004. The southern Araçuaí belt and the Dom Silvério Group: geologic architecture and tectonic significance. Anais Acad. Bras. Ciêenc. 76, 771-790.

Piuzana, P., Pimentel, M.M., Fuck, R.A., Armstrong, R., 2003. Neoproterozoic granulite facies metamorphism and coeval granitic magmatism in the Brasilia Belt Central Brazil: regional implications of new SHRIMP U-Pb and Sm-Nd data. Precamb. Res. 125, 245-273.

Porada, H., 1989. Pan-African rifting and orogenesis in southern to equatorial Africa and Eastern Brazil. Precamb. Res. 44, 103-136.

Rogers, J.W., Santosh, M., 2004. Continents and Supercontinents. Oxford University Press, p. 289.

Silva, L.C., Armstrong, R., Noce, C.M, Carneiro, M.A., Pimentel, M.M., Pedrosa-Soares, A.C., Leite, C.A., Vieira, V.S., Silva, M.A., Paes, V.J.C., Cardoso-Filho, J.M., 2002. Reavaliação da evolução geológica em terrenos pré-cambrianos brasileiros com base em novos dados U-Pb SHRIMP. Parte II. Orógeno Araçuaí, Cinturão Mineiro e Cráton São Francisco Meridional. Rev. Bras. Geociênc. 32, 513-528.

Silva, L.C., McNaughton, N.J., Armstrong, R., Hartmann, L.A., Fletcher, I.R., 2005a. The Neoproterozoic Mantiqueira Province and its African connections: a zircon-based $\mathrm{U}-\mathrm{Pb}$ geochronologic subdivision for the Brasiliano/Pan-African systems of orogens. Precamb. Res. 136, 203-240.

Silva, L.C., McNaughton, N.J., Fletcher, I.R., 2005b. SHRIMP U-Pb zircon geochronology of Neoproterozoic crustal granitoids (Southern Brazil): A case for discrimination of emplacement and inherited ages. Lithos 82, 503-525.

Steiger, R.H., Jäger, E., 1977. Subcommision on geochronology convention on the use of decay constants in geo- and cosmochronology. Earth Planet. Sci. Lett. 36, 359-362.

Tack, L., Wingate, M.T.D., Liégeois, J.-P., Fernandez-Alonso, M., Deblond, A., 2001. Early Neoproterozoic magmatism (1000-910 Ma) of the Zadinian and Mayumbian Groups (BasCongo): onset of Rodinia rifting at the western edge of the Congo craton. Precamb. Res. 110, 277-306.

Teixeira, W., Sabaté, P., Barbosa, J.S.F., Noce, C.M., Carneiro, M.A., 2000. Archean and Paleoproterozoic tectonic evolution of the São Francisco Craton. In: Cordani, U.G., Milani, E.J., Thomaz-Filho, A, Campos, D.A. (Eds.), Tectonic Evolution of South America. 31st Intern. Geol. Congr., pp. 101-138.

Tikhomirova, M., 2002. Zircon inheritance in diatexite granodiorites and its consequence on geochronology - a case study in Lusatia and the Erzgebirge (Saxo-Thuringia, eastern Germany). Chem. Geol. 191, 209-224.

Trompette, R., 1994. Geology of Western Gondwana (2000-500 Ma). Pan-African-Brasiliano aggregation of South America and Africa. A.A. Balkema, Rotterdam, p. 350.

Vicat, J.-P., Pouclet, A., 2000. Paleo- and Neoproterozoic granitoids and rhyolites from the West Congolian Belt (Gabon, Congo, Cabinda, north Angola): chemical composition and geotectonic implications. J. African Earth Sci. 31, 597617.

Watson, E.B., Harrison, T.M., 1983. Zircon saturation revisited: temperature and composition effects in a variety of crustal magma types. Earth Planet. Sci. Lett. 64, 295-304.

Williams, I.S., 1998. U-Th-Pb geochronology by ion microprobe. In: McKibben, M.A., Sahnks, W.C., Ridley, W.I. (Eds.), Applications of Microanalytical Techniques to Understanding Mineralizing Processes. Rev. Econ. Geol. 7, 1-35.

Williams, I.S., Meyer, C., 1998. U-Pb geochronology of zircons from lunar breccia 73217 using a sensitive high mass-resolution ín microprobe. J. Geophys. Res. 89, 525-534. 